\title{
Development of an Advanced Torque Vectoring Control System for an Electric Vehicle with In-Wheel Motors using Soft Computing Techniques
}

\author{
Kiumars Jalali, Thomas Uchida, Steve Lambert and John McPhee \\ Univ. of Waterloo
}

\begin{abstract}
A two-passenger, all-wheel-drive urban electric vehicle (AUTO21EV) with four direct-drive in-wheel motors has been designed and developed at the University of Waterloo. A 14-degree-of-freedom model of this vehicle has been used to develop a genetic fuzzy yaw moment controller. The genetic fuzzy yaw moment controller determines the corrective yaw moment that is required to stabilize the vehicle, and applies a virtual yaw moment around the vertical axis of the vehicle. In this work, an advanced torque vectoring controller is developed, the objective of which is to generate the required corrective yaw moment through the torque intervention of the individual in-wheel motors, stabilizing the vehicle during both normal and emergency driving maneuvers. Novel algorithms are developed for the left-to-right torque vectoring control on each axle and for the front-to-rear torque vectoring distribution action. Several maneuvers are simulated to demonstrate the performance and effectiveness of the proposed advanced torque vectoring controller, and the results are compared to those obtained using the ideal genetic fuzzy yaw moment controller. The advanced torque vectoring controller is also implemented in a hardware- and operator-in-the-loop driving simulator to further evaluate its performance.
\end{abstract}

CITATION: Jalali, K., Uchida, T., Lambert, S. and McPhee, J., "Development of an Advanced Torque Vectoring Control System for an Electric Vehicle with In-Wheel Motors using Soft Computing Techniques," SAE Int. J. Alt. Power. 2(2):2013, doi:10.4271/2013-01-0698.

\section{INTRODUCTION}

The AUTO21EV vehicle model with four direct-drive inwheel motors and an active steering system is an exciting platform on which to apply advanced motion control techniques, such as advanced slip control and torque vectoring systems, since the motor speeds and torques can be generated and controlled quickly, precisely, and independently at each wheel. An advanced fuzzy slip control system is developed and evaluated in [9] using predefined test maneuvers and a novel path-following driver model [10]. In addition, a genetic fuzzy yaw moment controller is developed in [11], the objective of which is to determine the corrective yaw moment required to minimize the vehicle yaw rate and sideslip errors. This genetically-tuned fuzzy yaw moment controller acts as a high-level supervisory module that assigns tasks to the lower-level controllers and actuators. In this paper, an advanced torque vectoring controller is developed for the AUTO21EV that distributes the task of generating the calculated corrective yaw moment to the inwheel motors. The developed advanced torque vectoring controller consists of left-to-right and front-to-rear torque vectoring components, which work together to distribute the calculated corrective yaw moment in an integrated approach.

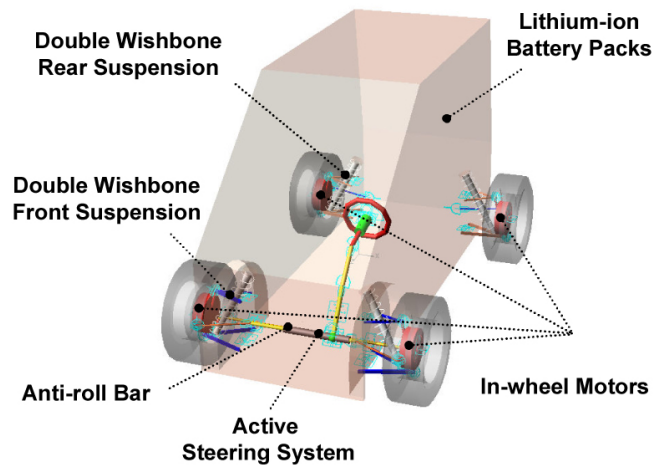

Figure 1. AUTO21EV concept vehicle

Figure 1 illustrates the AUTO21EV, which is a twopassenger, all-wheel-drive urban electric vehicle developed and modeled using the ADAMS/View software. Table 1 lists some of the relevant parameters used for the AUTO21EV model. The use of small but powerful direct-drive in-wheel motors allows for the implementation of the most advanced 
all-wheel-drive system in which the optimal traction force can be generated and controlled on each wheel.

Table 1. AUTO21EV model parameters

\begin{tabular}{|l|c|c|}
\hline Parameter & Value & Unit \\
\hline Vehicle mass $(M)$ & 1075 & $\mathrm{~kg}$ \\
\hline Vehicle yaw moment of inertia $\left(\mathrm{I}_{\mathrm{zz}}\right)$ & 1171 & $\mathrm{~kg} \mathrm{~m}$ \\
\hline Distance from CG to front axle $(\mathrm{a})$ & 0.82 & $\mathrm{~m}$ \\
\hline Distance from CG to rear axle $(\mathrm{b})$ & 0.98 & $\mathrm{~m}$ \\
\hline Front track width $\left(\mathrm{t}_{\mathrm{f}}\right)$ & 1.275 & $\mathrm{~m}$ \\
\hline Rear track width $\left(\mathrm{t}_{\mathrm{r}}\right)$ & 1.35 & $\mathrm{~m}$ \\
\hline Front tire cornering stiffness $\left(\mathrm{C}_{\mathrm{a}} \mathrm{F}\right)$ & 45570 & $\mathrm{~N} / \mathrm{rad}$ \\
\hline Rear tire cornering stiffness $\left(\mathrm{C}_{\mathrm{a} \mathrm{R}}\right)$ & 45570 & $\mathrm{~N} / \mathrm{rad}$ \\
\hline Tire size & $175 / 55 \mathrm{R} 15$ & - \\
\hline Tire unloaded radius $(\mathrm{r})$ & 0.29 & $\mathrm{~m}$ \\
\hline
\end{tabular}

\section{TORQUE VECTORING CONTROL SYSTEMS}

In conventional four-wheel-drive (4WD) vehicles, either all the wheels of the vehicle are permanently driven, which is referred to as an all-wheel-drive (AWD) vehicle, or one of the two axles is always linked to the engine and the second can be engaged (either manually or automatically) when needed. In both cases, in order to transmit the available engine torque to the wheels, inter-axle differentials or clutches must be installed on the propshaft, between the front and rear axles, and on both drive axles in order to split the torque between the left and right wheels. A comprehensive overview of the drivelines and differentials available for 4WD and AWD vehicles can be found in $[\underline{1}, \underline{2}]$. Although these powertrain configurations can enhance the traction and driving dynamics when faced with various road conditions, they suffer from higher costs, weight, and power consumption, as well as inefficiency.

In recent years, the market for AWD vehicles has evolved and expanded, and customer expectations of the driving dynamics and stability of such vehicles have increased dramatically. In addition, consumers are increasingly willing to request AWD on their new cars at extra cost []] . In order to meet these requirements and remain competitive in the market, active differentials for driveline systems are being introduced, which are able to distribute the engine torque to the front and rear axles, as well as to the left and right wheels on each axle, depending on the driving maneuver and road conditions. The possibility of actively influencing the distribution of the available engine torque based on the driving situation and traction conditions has led to an upheaval of the AWD market [4]. However, pressures to reduce $\mathrm{CO}_{2}$ emissions and increase fuel efficiency standards force the weight, cost, and power requirements of these actuators to be minimized.

Torque vectoring is the term introduced by the Ricardo Company [2] to describe a means of varying the distribution of engine torque between two outputs of a differential unit by controlling the torque over a relatively small speed difference between the outputs. Torque is directed in proportion to the relative shaft speeds, and can be biased seamlessly from one output to the other. Active torque vectoring systems were later introduced into the powertrain system, presenting torque-on-demand capabilities, where a center differential distributes the available engine torque to the front and rear axle differentials. Each axle differential can be equipped with an active torque vectoring system as well, which can be used to distribute the torque between the left and right wheels. An active center differential apportions the torque depending on the driving dynamics and the traction potentials on each axle, whereas an active axle differential distributes the torque based on the vehicle dynamics and the traction potential on each wheel of a single axle.

The benefits of active torque vectoring technology are twofold: the enhancement of vehicle traction and the enhancement of vehicle stability. On the one hand, torque vectoring can be used to modulate the individual wheel speeds to keep each tire operating in its optimal longitudinal slip range for the best traction. On the other hand, torque vectoring can be used to enhance the vehicle handling response by generating a corrective yaw moment to influence the vehicle yaw behavior []․ For instance, Figure 2 illustrates an active powertrain with an active center differential, an active rear differential, and an open front differential [6]. In normal driving conditions, where the differential actuators are not active, the center differential splits the torque generated by the engine in a fixed ratio of 40:60 percent to the front and rear axles, respectively, using a planetary gear. This fixed torque distribution ratio can be established based on the axle-load ratios, the design philosophy of the vehicle, or the desired handling characteristics of the vehicle [ 8$]$.

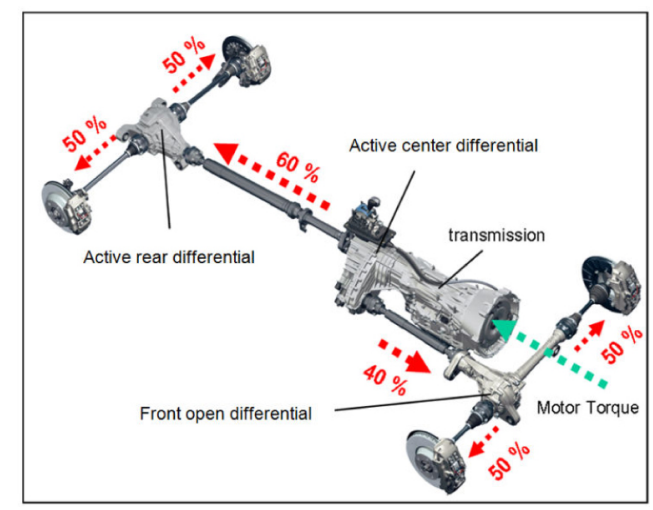

Figure 2. An active powertrain system with active center and rear differentials [6]

In addition, the front and rear differentials split their received torques in half using open differentials. Using torque vectoring technology for traction enhancement, the torque vectoring ratios on the center and rear differentials can vary depending on the traction potentials available on each axle or 


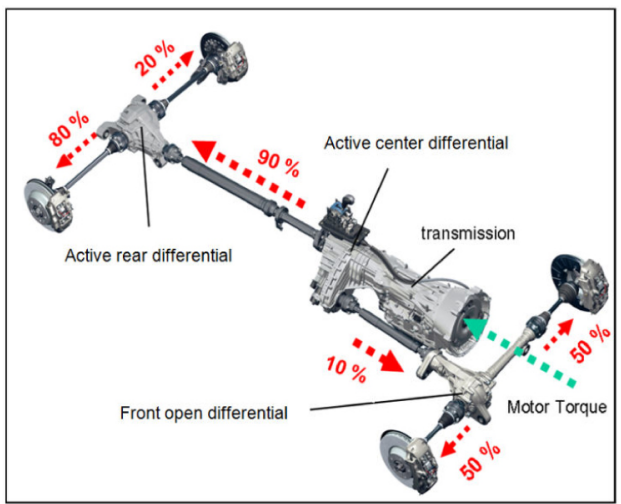

Figure 3. Torque vectoring in an active powertrain to enhance the vehicle traction [6]

tire, such that the highest traction force on each wheel is attained. Figure 3 illustrates the case where the front tires are on ice with almost no traction, and more traction is available on the rear-right tire than the rear-left tire. As can be seen, the active center differential is capable of sending 90 percent of the available engine torque to the rear axle, where more traction is available. Meanwhile, the active rear differential splits the torque received from the center differential asymmetrically by a ratio of 20:80 percent for the left and right wheels of the rear axle, respectively.

Torque vectoring can also be used as a stability control system, where the available drive torque is distributed among the wheels in order to generate a corrective yaw moment about the vertical axis of the vehicle. The corrective yaw moment can be generated in a left-to-right torque vectoring mode, where the available drive torque is distributed asymmetrically to the left and right wheels of an axle, or in a front-to-rear mode, where the available engine torque is distributed asymmetrically to the front and rear axles. For instance, an oversteering situation, in which the adhesion potential has been reached at the rear axle, can be corrected using left-to-right torque vectoring on the front axle, where more torque is transmitted to the front-inside tire (Figure 4-a). Conversely, an understeering situation, in which the adhesion potential has been exceeded on the front axle, can be corrected using left-to-right torque vectoring on the rear axle, where a higher percentage of the available axle torque is transmitted to the rear-outside tire in order to generate the required corrective yaw moment (Figure 4-b)

The driving dynamics of a vehicle can also be influenced by varying the front-to-rear torque distribution. The front-torear torque vectoring mode takes advantage of the interconnection between the longitudinal and lateral tire forces, where changing one force will automatically influence the other. For instance, an understeered vehicle can be controlled by transmitting more of the engine torque to the rear axle than the front axle. This strategy not only increases the lateral force potential at the front axle and, therefore, enhances the ability of the driver to steer the vehicle, but it also reduces the lateral force potential at the rear axle by increasing the longitudinal traction force. The lateral force difference between the two axles generates the required corrective yaw moment about the vertical axis of the vehicle, helping the vehicle to travel in the direction in which it is pointed (Figure 5-a). Conversely, an oversteered vehicle can be controlled by transmitting more of the available engine torque to the front axle, thereby increasing the essential lateral force potential on the rear axle. At the same time, this strategy will reduce the lateral force potential on the front axle by elevating its longitudinal force, thus helping the vehicle to develop more understeering behavior (Figure 5-b).

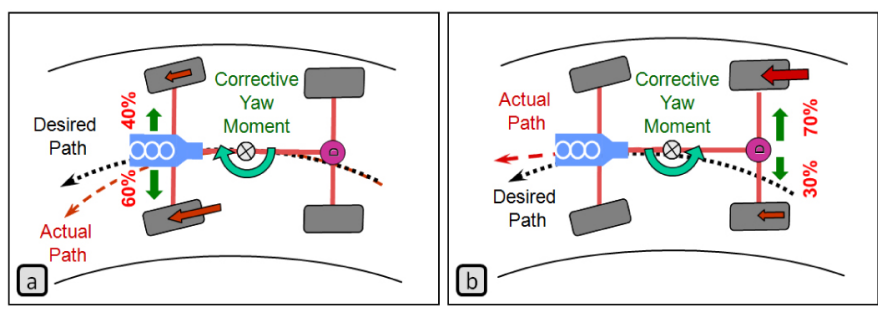

Figure 4. (a) Stability control of an oversteered vehicle through left-to-right torque vectoring on the front axle, and (b) stability control of an understeered vehicle through left-to-right torque vectoring on the rear axle
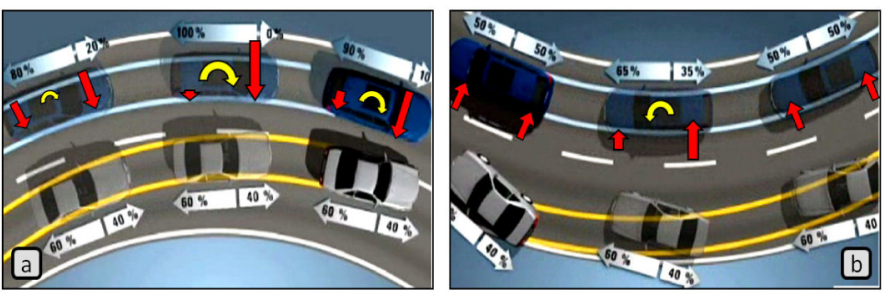

Figure 5. Front-to-rear torque vectoring (a) in an understeered vehicle, and (b) in an oversteered vehicle [8]

Although the theory behind the torque vectoring technique is similar to that used for an electronic stability control (ESC) system, torque vectoring is more effective, especially at generating a corrective yaw moment at higher 
vehicle speeds and during emergency maneuvers near the handling limits of the vehicle [5]. In general, torque vectoring can affect the vehicle driving dynamics and traction while causing almost no change in the total driving force of the vehicle. Active braking, on the other hand, is hampered by a net braking effect and drive torque reduction, which not only increases the inefficiency of the vehicle by eliminating power that has already been produced, but it has also been reported by drivers as being disruptive due to the unexpected speed reduction. Moreover, in contrast to active braking, which is allowed only a limited operation time to ensure a safe reserve of fade-free braking performance, torque vectoring can be employed much more actively to enhance driving dynamics and vehicle traction even in normal driving conditions to improve the everyday driving experience. However, it is important to note that a torque vectoring system can be effective only when a driving torque exists in the first place. In other words, torque vectoring is not able to intervene when the driver releases the throttle or brakes the vehicle. In such cases, an ESC system must maintain the stability of the vehicle. Therefore, torque vectoring and active braking should be considered to be complementary technologies whose full potential can be realized only if a holistic integrated approach is used to operate both systems under a common supervisory controller.

In summary, to control the vehicle traction and driving dynamics during both braking and acceleration maneuvers, the vehicle should be equipped with an antilock braking system (ABS), a traction control system (TCS), ESC, and torque vectoring control systems, and all of these systems should be networked together in an integrated fashion. However, equipping a vehicle with all the aforementioned control systems and actuators is a very expensive and complex task. It is for this reason that such a degree of vehicle control is currently available in only luxury-class vehicles, where the customer is prepared to pay for these technologies.

\section{CONTROL METHOD FOR LEFT-TO- RIGHT TORQUE VECTORING DISTRIBUTION}

The objective of the advanced torque vectoring system is to distribute the calculated corrective yaw moment from the high-level yaw moment controller to the individual in-wheel motors in order to stabilize the vehicle driving dynamics. Assuming that $M_{z}$ is the total required corrective yaw moment that is calculated by the genetic fuzzy yaw moment controller, the tire forces on each axle must be adjusted such that each axle generates a portion of the total corrective yaw moment:

$$
M_{z}=M_{z, \text { front }}+M_{z, \text { rear }}=\theta_{f} \cdot M_{z}+\theta_{r} \cdot M_{z}
$$

where $M_{z, \text { front }}$ and $M_{z \text {,rear }}$ are the portions of the required corrective yaw moment that must be generated at the front and rear axles, respectively. In other words, $\theta_{f}$ and $\theta_{r}$ are the percentages of the total required corrective yaw moment $M_{z}$ that must be generated at the front and rear axles, such that $\theta_{f}$ $+\theta_{r}=100 \%$. The relationship between $\theta_{f}$ and $\theta_{r}$ defines the front-to-rear torque vectoring distribution, and will be discussed below.

Generating a couple (equal and opposite traction forces) on each axle is the best strategy for creating the required corrective yaw moment for two reasons: first, all tires participate in generating the required corrective yaw moment; second, the vehicle speed will not be influenced by the activities of the torque vectoring system as they would in an ESC system, provided the forces on the sides of each axle can be generated without being restricted by the traction potential of the tires or the performance of the in-wheel motors. In addition, since all four tires are involved in generating the corrective yaw moment, and since each tire can be accelerated or braked independently, this strategy is more efficient than conventional ESC and torque vectoring systems, where only some tires are involved in generating the corrective yaw moment. Figure 6 illustrates the proposed torque vectoring strategy, where a couple is generated on each axle. The generated yaw moment on each axle is calculated as follows:

$$
\begin{aligned}
& \left.M_{z, \text { front }}=F_{x, F R} \cdot \frac{t_{f}}{2}+F_{x, F L} \cdot \frac{t_{f}}{2}\right\} \Rightarrow \\
& F_{x, F R}=F_{x, F L}=F_{x, F} \\
& M_{z, \text { front }}=F_{x, F} \cdot t_{f} \Rightarrow F_{x, F}=\frac{M_{z, \text { front }}}{t_{f}} \\
& \left.\begin{array}{l}
M_{z, \text { rear }}=F_{x, R R} \cdot \frac{t_{r}}{2}+F_{x, R L} \cdot \frac{t_{r}}{2} \\
F_{x, R R}=F_{x, R L}=F_{x, R}
\end{array}\right\} \Rightarrow \\
& M_{z, \text { rear }}=F_{x, R} \cdot t_{r} \Rightarrow F_{x, R}=\frac{M_{z, \text { rear }}}{t_{r}}
\end{aligned}
$$

where $F_{x, F R}, F_{x, F L}, F_{x, R R}$, and $F_{x, R L}$ are the longitudinal tire forces of the front-right, front-left, rear-right, and rear-left wheels, respectively; $t_{f}$ and $t_{r}$ are the front and rear wheel track widths.

In order to calculate the required motor torques, a torque balance is formed for each wheel. Figure 7 illustrates the model of a single wheel, where $I_{y y, w}$ denotes the moment of inertia of the wheel about its spin axis, $T_{m}$ denotes the motor torque, $r_{d y n}$ is the tire dynamic radius, $\omega_{w}$ is the angular velocity of the tire, $F_{z}$ is the tire vertical force, and $F_{x}$ is the tire longitudinal force. Note that the tire rolling resistance and the aerodynamic drag of the vehicle are neglected in this 
model for simplicity. By specifying the equation of motion of the wheel, the traction force at the tire-road contact patch can be estimated as follows:

$$
T_{m}-F_{x} \cdot r_{d y n}=I_{y y, w} \cdot \dot{\omega}_{w} \Leftrightarrow F_{x}=\frac{1}{r_{d y n}} \cdot\left(T_{m}-I_{y y, w} \cdot \dot{\omega}_{w}\right)
$$

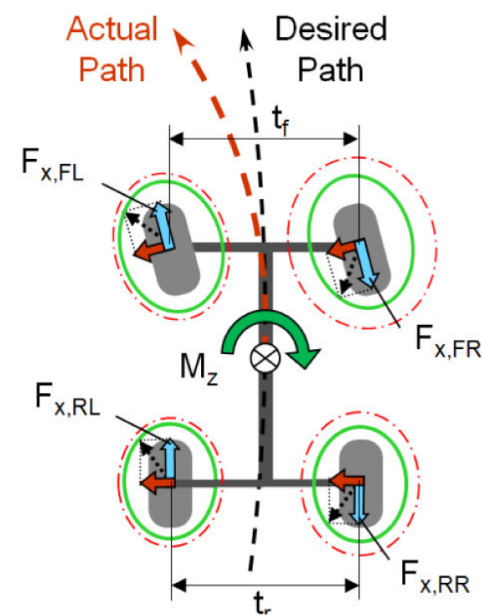

Figure 6. Advanced torque vectoring control strategy using couple generation on each axle (the dash-dotted ellipse surrounding each tire indicates the adhesion potential of that tire; the solid ellipse indicates the actual friction ellipse)

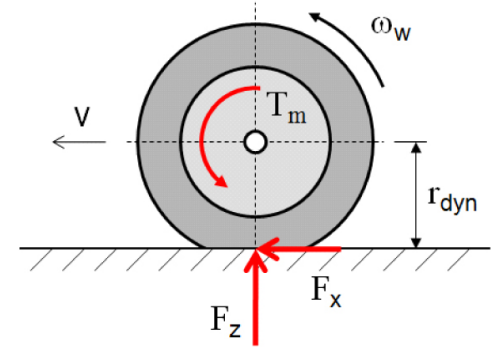

Figure 7. Torque balance at the tire-road contact patch

Substituting the traction force $F_{x}$ from equation (4) into equations (2) and (3), the required motor torque at each wheel can be calculated as follows:

$$
T_{m, i}=\frac{r_{d y n, i}}{t_{f}} \cdot M_{z, \text { front }}+I_{y y, w} \cdot \dot{\omega}_{w, i}
$$

where $\mathrm{i} \in\{\mathrm{FR}, \mathrm{FL}\}$

$$
T_{m, i}=\frac{r_{d y n, i}}{t_{r}} \cdot M_{z, \text { rear }}+I_{y y, w} \cdot \dot{\omega}_{w, i}
$$

where $\mathrm{i} \in\{\mathrm{RR}, \mathrm{RL}\}$
Note that when the required corrective yaw moment is positive, the wheels on the right side of the vehicle must be driven and the wheels on the left side must be braked; when the required corrective yaw moment is negative, the wheels on the right side of the vehicle must be braked and those on the left side must be driven.

\section{CALCULATION OF TIRE ADHESION POTENTIAL}

To prevent the tires from spinning out or locking up during the couple generation, the maximum possible traction force of each tire is estimated at each time step of the simulation, and is used as a limit in the controller. In addition, the adhesion potential of each tire is calculated and used to define the extent to which the tire forces have been saturated. Estimating the adhesion potential requires information about the horizontal and vertical forces acting on the tire, as well as the friction coefficient between the tire and the road, the estimation of which is discussed below.

The longitudinal and lateral tire forces are estimated using the well-known "Magic Tire Formula" $[\underline{4}, \underline{5}]$. This model is a semi-empirical set of curve fits that takes into account the coupling between the longitudinal and lateral tire forces through combined-slip characteristics, a limited tire adhesion potential, the variation in cornering stiffness with tire load, and the influence of the tire-road friction coefficient. The combined-slip horizontal tire forces are estimated using the following equations [12]:

$$
\begin{aligned}
& F_{x, i}=F_{x o, i} \cdot \cos \left(C \cdot \arctan \left(B \cdot \alpha_{i}-E \cdot\left(B \cdot \alpha_{i}-\arctan \left(B \cdot \alpha_{i}\right)\right)\right)\right) \\
& F_{y, i}=F_{y o, i} \cdot \cos \left(C \cdot \arctan \left(B \cdot \lambda_{i}-E \cdot\left(B \cdot \lambda_{i}-\arctan \left(B \cdot \lambda_{i}\right)\right)\right)\right)
\end{aligned}
$$

where $i \in\{\mathrm{FL}, \mathrm{FR}, \mathrm{RL}, \mathrm{RR}\}$. Note that these forces are calculated with respect to the wheel coordinate system. $F_{x o, i}$ and $F_{y o, i}$ represent the pure-slip tire forces in the longitudinal and lateral directions, respectively, and are calculated as follows [12]:

$$
\begin{gathered}
F_{x o, i}=D \cdot \sin \left(C \cdot \arctan \left(B \cdot \lambda_{i}-E \cdot\left(B \cdot \lambda_{i}-\arctan \left(B \cdot \lambda_{i}\right)\right)\right)\right) \\
F_{y o, i}=D \cdot \sin \left(C \cdot \arctan \left(B \cdot \alpha_{i}-E \cdot\left(B \cdot \alpha_{i}-\arctan \left(B \cdot \alpha_{i}\right)\right)\right)\right)
\end{gathered}
$$

In this tire model, the interdependence between the longitudinal and lateral tire forces is considered, where peak factors $D$, shape factors $C$, stiffness factors $B$, and curvature factors $E$ are different for equations (7),(8),(9),(10), and for the longitudinal and lateral directions [12]. All the parameters required by this model are taken from the Pacejka 2002 tire data obtained for a 175/55 R15 tire. In addition, the slip ratio $(\lambda)$ of each tire is calculated using equations (1) and (2) in [9], 
and the sideslip angle $(\alpha)$ of each tire is approximated using a bicycle model, as indicated below [14]:

$$
\begin{gathered}
\alpha_{F}=\delta+\beta-\frac{a \cdot \dot{\psi}}{v_{x}} \\
\alpha_{R}=\beta+\frac{b \cdot \dot{\psi}}{v_{x}}
\end{gathered}
$$

where $\delta$ is the steering angle, $\beta$ is the sideslip angle of the vehicle, $a$ and $b$ are the distances of the front and rear axles from the vehicle center of gravity, $v_{x}$ is the vehicle forward speed, and $\dot{\psi}$ is the vehicle yaw rate.

Approximating the longitudinal and lateral tire forces using equations (7) and (8), the tire adhesion potential utilization $\eta$ can be estimated using the following elliptic relation:

$$
\begin{aligned}
& \eta_{i}=\sqrt{\left(\frac{F_{x i}}{F_{x i, \max }}\right)^{2}+\left(\frac{F_{y i}}{F_{y i, \max }}\right)^{2}}, \\
& 0 \leq \eta_{i} \leq 1 \text { and } i \in\{\mathrm{FL}, \mathrm{FR}, \mathrm{RL}, \mathrm{RR}\}
\end{aligned}
$$

where $F_{x i \text {,max }}$ and $F_{y i \text { max }}$ represent the maximum possible forces in the longitudinal and lateral directions, respectively. With the nominal tire load $F_{z 0}$ and the tire-road friction coefficients $\mu_{i}$, the maximum longitudinal and lateral tire forces can be approximated as follows [13]:

$$
\begin{aligned}
& F_{x i, \max }=\mu_{i} \cdot F_{z i} \cdot k_{x, i} \cdot\left(1+l_{x} \cdot \frac{F_{z i}-F_{z 0}}{F_{z 0}}\right) \\
& F_{y i \text {,max }}=\mu_{i} \cdot F_{z i} \cdot k_{y, i} \cdot\left(1+l_{y} \cdot \frac{F_{z i}-F_{z 0}}{F_{z 0}}\right)
\end{aligned}
$$

where $k_{x, i}$ and $k_{y, i}$ depend on the actual tire camber angle, and parameters $l_{x}$ and $l_{y}$, which define the degressive behavior of the tire horizontal forces [12], are set to 1 . For the sake of simplicity, the camber angles in the ADAMS/View model of the AUTO21EV are assumed to be zero and constant throughout the simulation.

The actual vertical force applied to each tire $\left(F_{z i}\right)$ is approximated by neglecting the coupling between the vehicle roll and pitch motions, and by disregarding the suspension dynamics. To this end, two half-car models are defined, one for the longitudinal direction and one for the lateral direction. Figure 8-a illustrates a half-car model in the longitudinal direction, where $a_{x}$ indicates the longitudinal acceleration of the vehicle. The inertial force due to the longitudinal acceleration at the vehicle center of gravity $(\mathrm{CG})$ causes a weight shift to the rear axle, which simultaneously reduces the front axle load and increases the rear axle load. Constructing the torque balance at the rear axle contact point yields the following expression for the front axle load $\left(F_{z, F}\right)$ [15]:

$$
\begin{aligned}
& \sum M_{y}=0 \Rightarrow \\
& (a+b) \cdot F_{z, F}-m_{C G} \cdot g \cdot b+m_{C G} \cdot a_{x} \cdot h_{C G}=0 \Rightarrow \\
& F_{z, F}=\frac{b}{a+b} m_{C G} \cdot g-\frac{h_{C G}}{a+b} m_{C G} \cdot a_{x}
\end{aligned}
$$

where $a$ and $b$ are the distances of the front and rear axles from the vehicle center of gravity, $m_{C G}$ is the total mass of the vehicle, $h_{C G}$ is the height of the vehicle center of gravity, and $g$ is the gravitational acceleration constant. $a_{x}$ is the longitudinal acceleration of the vehicle, and can be measured using an accelerometer. Subtracting the front axle load $\left(F_{z, F}\right)$ from the total vehicle weight, the rear axle load $\left(F_{z, R}\right)$ is calculated as follows:

$$
\begin{aligned}
& \sum F_{z}=0 \Rightarrow \mathrm{F}_{z, F}+\mathrm{F}_{z, R}-m_{C G} \cdot g=0 \Rightarrow \\
& \mathrm{F}_{z, R}=\frac{a}{a+b} m_{C G} \cdot g+\frac{h_{C G}}{a+b} m_{C G} \cdot a_{x}
\end{aligned}
$$
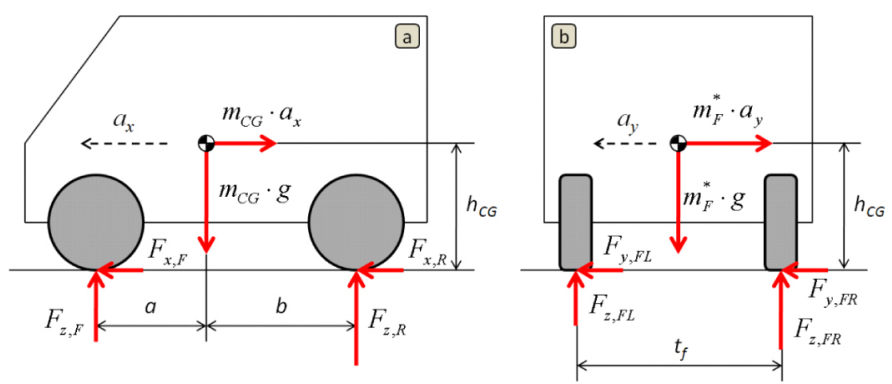

Figure 8. (a) Longitudinal weight shift during acceleration, and (b) lateral weight shift during cornering

During cornering, the lateral acceleration causes a weight shift to one side of each axle, whose distribution between the front and rear axles depends on the axle loads. The two axles are considered to be decoupled from each other, and a halfcar model is used to calculate the lateral weight shift on each axle. For instance, Figure 8-b illustrates the half-car model for the front axle, where the virtual mass of the front axle $\left(m_{F}^{*}\right)$ is calculated as follows: 


$$
m_{F}^{*}=\frac{F_{z, F}}{g}=\frac{F_{z, F L}+F_{z, F R}}{g}
$$

Considering the torque balance equation at the point of contact between the ground and the front-left tire, the lateral weight shift at the front-right tire is calculated as follows:

$$
\begin{aligned}
& \sum M_{x}=0 \Rightarrow \\
& F_{z, F R} \cdot t_{f}-m_{F}^{*} \cdot a_{y} \cdot h_{C G}-m_{F}^{*} \cdot g \cdot \frac{t_{f}}{2}=0 \Rightarrow \\
& F_{z, F R}=\frac{m_{F}^{*}}{t_{f}}\left(a_{y} \cdot h_{C G}+\frac{t_{f}}{2}\right)
\end{aligned}
$$

Substituting into equation (19) the virtual mass of the front axle $\left(m_{F}^{*}\right)$ from equation (18) and the front axle load $\left(F_{z, F}\right)$ from equation (16), the front-right dynamic wheel load can be calculated as follows:

$$
F_{z, F R}=m_{C G} \cdot\left(\frac{b}{a+b} g-\frac{h_{C G}}{a+b} a_{x}\right) \cdot\left[\frac{1}{2}+\frac{h_{C G} \cdot a_{y}}{t_{f} \cdot g}\right]
$$

The dynamic loads of the other three wheels can be computed by analogy:

$$
\begin{aligned}
& F_{z, F L}=m_{C G} \cdot\left(\frac{b}{a+b} g-\frac{h_{C G}}{a+b} a_{x}\right) \cdot\left[\frac{1}{2}-\frac{h_{C G} \cdot a_{y}}{t_{f} \cdot g}\right] \\
& F_{z, R R}=m_{C G} \cdot\left(\frac{a}{a+b} g+\frac{h_{C G}}{a+b} a_{x}\right) \cdot\left[\frac{1}{2}+\frac{h_{C G} \cdot a_{y}}{t_{r} \cdot g}\right] \\
& F_{z, R L}=m_{C G} \cdot\left(\frac{a}{a+b} g+\frac{h_{C G}}{a+b} a_{x}\right) \cdot\left[\frac{1}{2}-\frac{h_{C G} \cdot a_{y}}{t_{r} \cdot g}\right]
\end{aligned}
$$

Note that the performance and accuracy of this method have been proven in [15] by comparing data measured during a severe cornering maneuver with the wheel loads approximated by equations (20),(22),(23). Finally, substituting the traction force $\left(F_{x}\right)$ from equation (4) into equation (14), the maximum motor torque at each wheel can be calculated:

$$
\begin{aligned}
& \frac{1}{r_{d y n, i}} \cdot\left(T_{m, i_{\max }}-I_{y y, w_{i}} \cdot \dot{\omega}_{w_{i}}\right)=\mu_{i} \cdot F_{z i} \cdot k_{x, i} \cdot\left(1+l_{x} \cdot \frac{F_{z i}-F_{z 0}}{F_{z 0}}\right) \Rightarrow \\
& T_{m, i_{\max }}=r_{d y n, i} \cdot \mu_{i} \cdot F_{z i} \cdot k_{x, i} \cdot\left(1+l_{x} \cdot \frac{F_{z i}-F_{z 0}}{F_{z 0}}\right)+I_{y y, w_{i}} \cdot \dot{\omega}_{w_{i}}
\end{aligned}
$$

As can be seen, the maximum motor torque in equation (24) is a function of the tire-road friction coefficient $(\mu)$. Consequently, a friction coefficient estimator must ultimately be included in the advanced torque vectoring controller so that the maximum possible motor torque can be calculated correctly when driving in all road conditions. Equation (24) is used by the torque vectoring controller to limit the motor torque at each wheel when generating a couple.

In summary, by comparing equations (5) and (6) with equation (24), the motor torque at each wheel is limited as follows:

$$
T_{m, i}=\min \left[\begin{array}{l}
\left(\frac{r_{d y n, i}}{t_{f}} \cdot M_{z, f r o n t}+I_{y y, w} \cdot \dot{\omega}_{w, i}\right), \\
\left(r_{d y n, i} \cdot \mu_{i} \cdot F_{z i} \cdot k_{x, i} \cdot\left(1+l_{x} \cdot \frac{F_{z i}-F_{z 0}}{F_{z 0}}\right)+I_{y y, w_{i}} \cdot \dot{\omega}_{w_{i}}\right)
\end{array}\right]
$$

where $i \in\{\mathrm{FL}, \mathrm{FR}\}$ and

$$
T_{m, i}=\min \left[\begin{array}{l}
\left(\frac{r_{d y n, i}}{t_{r}} \cdot M_{z, \text { rear }}+I_{y y, w} \cdot \dot{\omega}_{w, i}\right), \\
\left(r_{d y n, i} \cdot \mu_{i} \cdot F_{z i} \cdot k_{x, i} \cdot\left(1+l_{x} \cdot \frac{F_{z i}-F_{z 0}}{F_{z 0}}\right)+I_{y y, w_{i}} \cdot \dot{\omega}_{w_{i}}\right)
\end{array}\right]
$$

where $i \in\{\mathrm{RL}, \mathrm{RR}\}$. As indicated in equation (1), $M_{z \text {,front }}$ and $M_{z, \text { rear }}$ are the portions of the required corrective yaw moment that must be generated at the front and rear axles, respectively. The relationship between these two moments defines the front-to-rear torque vectoring distribution, and will be defined in the next section.

\section{CONTROL METHOD FOR FRONT- TO-REAR TORQUE VECTORING DISTRIBUTION}

As mentioned earlier, if a center differential were used to distribute the driving torque between the front and rear axles, the fixed torque distribution could be established on the basis of the axle-load ratio, the design philosophy of the vehicle, or the desired handling characteristics of the vehicle. In the case of an active differential, however, this ratio can be adjusted according to the traction conditions or driving dynamics of the vehicle [7]. Since no mechanical linkage exists between the wheels of the AUTO21EV, the front-to-rear torque distribution ratio must be set virtually and adjusted based on the vehicle driving dynamics or traction conditions. In normal driving conditions, a fixed 50:50 ratio has been chosen to split the required corrective yaw moment equally between the front and rear axles. However, this ratio will be adjusted by a yaw rate feedback controller at high maneuvering speeds and in emergency situations where the vehicle is operating near its handing limits. This approach uses the yaw rate error calculated by the genetic fuzzy yaw moment controller 
(YMC) [11] and sets the front-to-rear distribution ratios, namely $\theta_{f}$ and $\theta_{r}$, such that the deviation between the desired and actual yaw rates of the vehicle is minimized. This objective is accomplished by a proportional-derivative (PD) controller as follows:

$$
\begin{gathered}
\theta_{f}=\theta_{f, \text { fixed }}+K_{P} \cdot e(\dot{\psi})+K_{D} \cdot \frac{d}{d t} e(\dot{\psi}) \\
\theta_{r}=100 \%-\theta_{f}
\end{gathered}
$$

where $\theta_{f \text {, fixed }}$ is the predefined fixed ratio of $50 \%$, and $K_{P}$ and $K_{D}$ are the proportional and derivative feedback gains of the PD controller, respectively. Note that, in this work, the controller gains are chosen manually through a trial-and-error approach. The performance and stability of this controller have been examined and tuned through numerous driving maneuvers in the simulation environment. Since the proposed PD controller is part of a digital control system, the derivative component of the controller is approximated as follows:

$$
\frac{d}{d t} e(\dot{\psi}) \approx \frac{e(\dot{\psi})_{k}-e(\dot{\psi})_{k-1}}{\text { sample time }}
$$

where $e(\dot{\psi})_{k}$ and $e(\dot{\psi})_{k-1}$ are the current and previous values of the yaw rate error, respectively.

\section{EVALUATION OF THE ADVANCED TORQUE VECTORING CONTROLLER}

In order to evaluate the performance of the advanced torque vectoring controller (ATVC), the AUTO21EV is first driven through a series of test maneuvers using an advanced path-following driver model [10].

\section{ISO Double-Lane-Change Maneuver}

The performance of the ATVC is first evaluated by driving the AUTO21EV through a double-lane-change maneuver with an initial speed of $75 \mathrm{~km} / \mathrm{h}$ and using a pathfollowing driver model [10]. Figure 9 illustrates the vehicle trajectory and demonstrates that the driver is able to negotiate the maneuver when the ATVC is active.

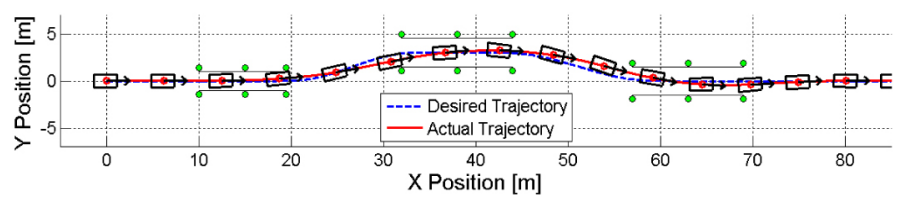

Figure 9. Desired and actual vehicle trajectories when driving through the double-lane-change maneuver with an initial speed of $75 \mathrm{~km} / \mathrm{h}$ using the path-following driver model and the ATVC
Figure 10 illustrates the vehicle yaw rate and sideslip angle during this maneuver. As can be seen, the ATVC is not able to exactly match the actual vehicle yaw rate with the desired yaw rate calculated using the reference bicycle model. In addition, due to the actuation of the in-wheel motors, there are some oscillations in the actual vehicle yaw rate that might be perceived by the driver as being annoying. However, the sideslip angle of the vehicle is very close to that obtained when the genetic fuzzy yaw moment controller (GFYMC) was active [11].
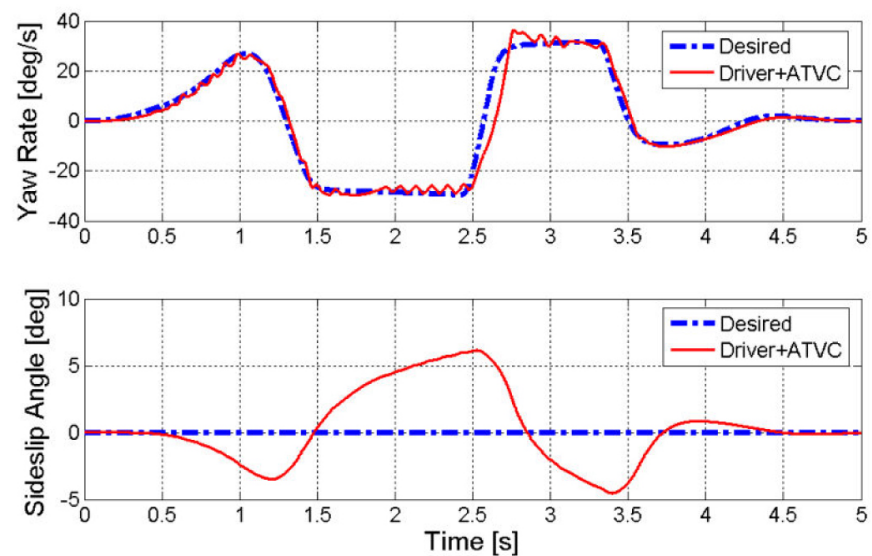

Figure 10. Desired and actual vehicle yaw rates (top) and sideslip angles (bottom) when driving through the double-lane-change maneuver using the driver model with and without the ATVC

Figure 11-a illustrates the vehicle lateral acceleration as a function of time. As can be seen, the results using the ATVC are very similar to those observed using the GFYMC except at the handling limits, where the activation of the in-wheel motors causes some oscillations in the lateral acceleration of the vehicle. As mentioned before, this type of oscillation might be perceived by the driver as being disruptive, and should be avoided. From Figure 11-b, it is confirmed that, except during the second lane change, the driver requires about the same amount of steering wheel input as is the case when the GFYMC is active. Figure 11-c illustrates the vehicle speed during the double-lane-change maneuver, and confirms the advantage of the couple generation by the ATVC. Specifically, due to the couples generated at the front and rear axles, the speed reduction during this maneuver is much smoother and more linear than is the case when no stability controller is active. As such, the vehicle loses almost the same amount of speed as when the ideal (but, of course, not directly realizable) GFYMC is active. Figure 11-d illustrates the handling performance of the vehicle and indicates that the hysteresis of this plot is twice as large as that of the analogous plot for the GFYMC. In other words, although the responsiveness and agility of the vehicle are considerably improved compared to the case where no stability controller is active, they are not as good as they are 
when the GFYMC is used to apply a corrective yaw moment directly to the vehicle CG.
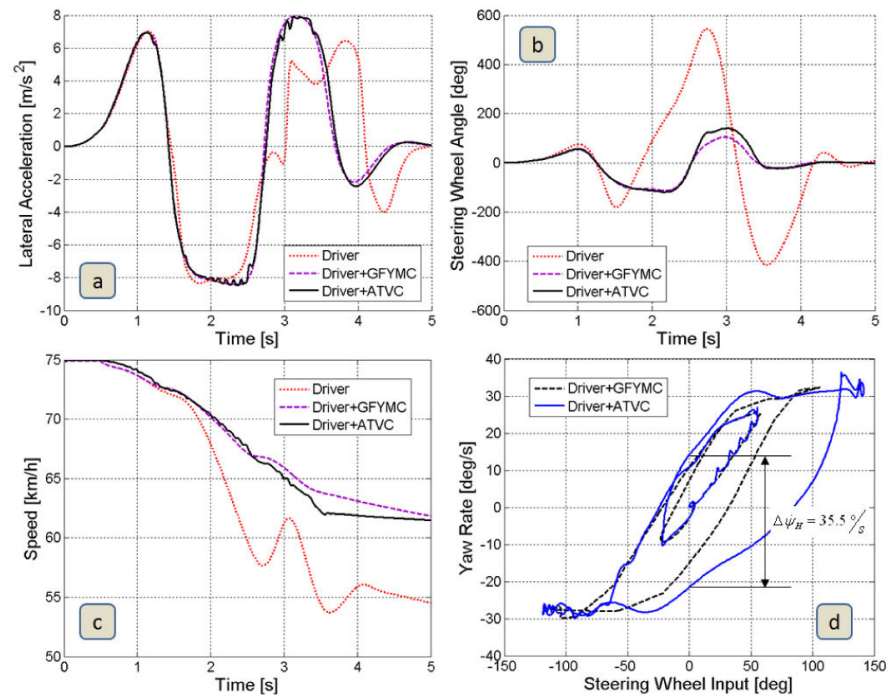

Figure 11. (a) Lateral acceleration, (b) steering wheel angle, and (c) vehicle speed as functions of time; and (d) vehicle yaw rate as a function of the steering wheel input when driving through the double-lane-change maneuver using the driver model without a controller, with the genetic fuzzy yaw moment controller (GFYMC), and with the ATVC

Figure 12 illustrates the torque of each in-wheel motor during the double-lane-change maneuver. It is very important to notice that, at each wheel, the requested motor torque from the torque vectoring controller can be restricted by the maximum possible motor torque as calculated in equation (24), the slip controller that prevents the tires from locking up or spinning out, or by the power limitations of the in-wheel motor. It is notable that the performance of the in-wheel motors decreases the faster the motors rotate due to inductive voltage losses. From the motor torque plots, it can be seen that, although the ATVC has always requested couple forces on the sides of both axles, the requested motor torques could not always be generated due to one of the aforementioned limitations. For instance, between 1.2 and 1.45 seconds of the simulation, the actual motor torques generated at the front wheels and the rear-right wheel are less than the requested torques because, at a speed of $72 \mathrm{~km} / \mathrm{h}$, the motors are not powerful enough to generate the requested torques. At the rear-left wheel, however, the requested motor torque is restricted by the maximum allowable torque. Another interesting region to observe is between 2.45 and 3.1 seconds of the simulation, during which time the transition between the minimum and maximum lateral accelerations occurs (Figure 11-b). As can be seen, the left-to-right torque vectoring controller has first requested the left wheels to brake and the right wheels to accelerate in order to correct an oversteering situation, where the actual yaw rate is larger than the desired one (Figure 10). At 2.73 seconds of the simulation, however, as the transition from a negative lateral acceleration to a positive one occurs and the yaw rate error becomes zero, the left-to-right torque vectoring controller changes its request by ordering the left wheels to accelerate and the right wheels to brake, which is again done to correct an oversteering situation.
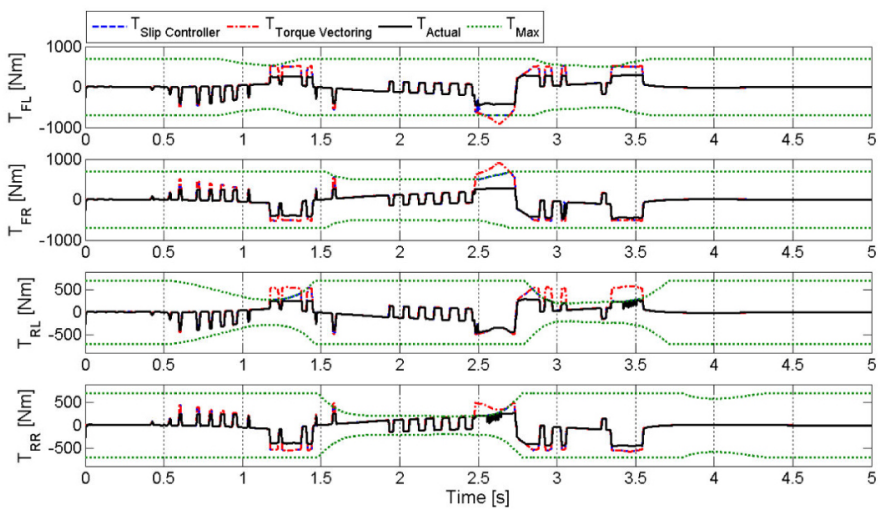

Figure 12. Requested and actual motor torques at each wheel when driving through the double-lane-change maneuver using the driver model with the ATVC

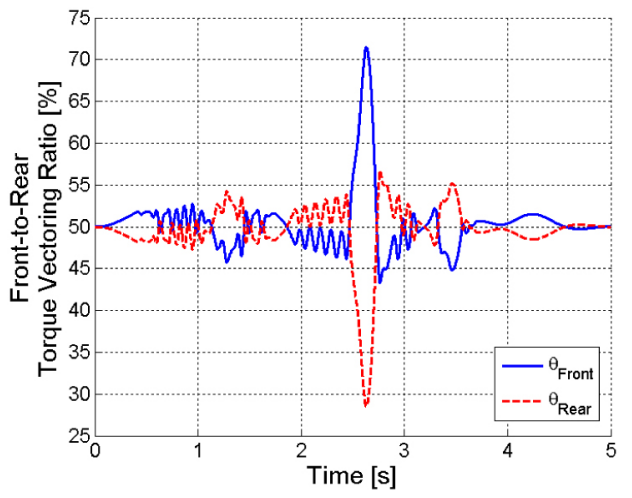

Figure 13. Front-to-rear torque vectoring ratio when driving through the double-lane-change maneuver using the driver model with the ATVC

From the activation history of the front-to-rear torque vectoring controller (Figure 13), it can be confirmed that the actions of the left-to-right torque vectoring controllers have been supported by the front-to-rear torque vectoring controller. In particular, the front-to-rear torque vectoring controller has requested the front motors to generate up to $72 \%$ of the total required corrective yaw moment between 2.45 and 2.73 seconds and, later, it changes its request by asking for more torque from the rear motors between 2.73 and 3.1 seconds of the simulation. Note that generating more torque at the front motors rather than the rear ones reduces the lateral force potential at the front axle and increases that at the rear axle. The asymmetric lateral force potentials on the front and rear axles is used to generate the required corrective yaw moment. The activity of the front-to-rear torque 
vectoring controller can also be seen in Figure 12. For instance, between 2.45 and 2.73 seconds, the front-to-rear torque vectoring controller has requested the front-left motor to generate up to $900 \mathrm{Nm}$ of braking torque and the frontright motor to generate up to $900 \mathrm{Nm}$ of driving torque while, at the same time, the controller has requested up to $350 \mathrm{Nm}$ of braking and driving torques from the rear-left and rearright motors, respectively. Note that, although the controller has requested the front motors to generate large motor torques, the motors are not powerful enough to generate the requested torques when the vehicle is traveling at a speed of $66 \mathrm{~km} / \mathrm{h}$. The effects seen in Figure 12 can also be confirmed by looking at the tire traction potentials and tire slip ratios shown in Figures 14 and $\underline{15}$, respectively. For instance, the maximum traction potential of the rear-left tire has been exceeded once at 1.2 seconds and then between 3.37 and 3.56 seconds of the simulation (Figure 14). From Figures 12 and $\underline{15}$, it can be confirmed that the requested motor torque on the rear-left wheel is first restricted by the maximum torque limiter (at 1.2 seconds) and then by the slip controller [9] (between 3.37 and 3.56 seconds) in order to prevent tire spinout. This observation suggests that the excess of the traction potential of the rear-left tire is due to the fact that the lateral force of that tire has exceeded its limit.
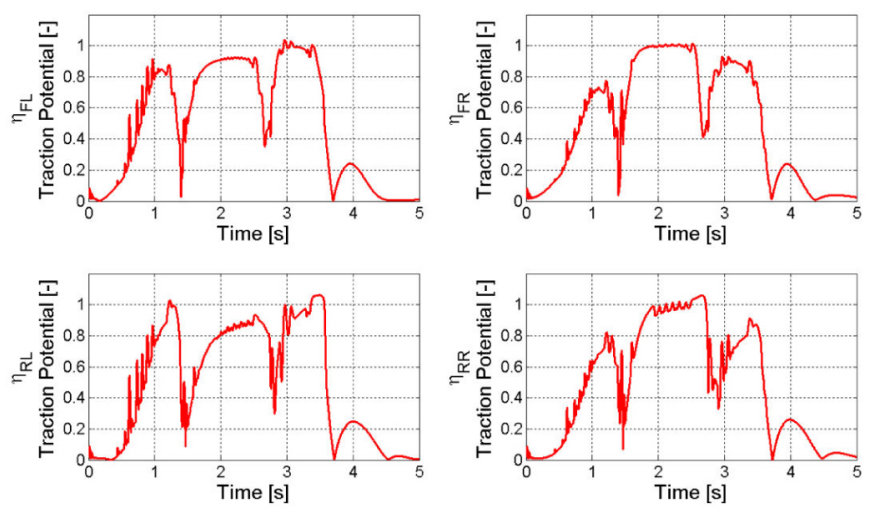

Figure 14. Traction potential of each tire when driving through the double-lane-change maneuver using the driver model with the ATVC

Table 2 summarizes the vehicle response during the double-lane-change maneuver using the path-following driver model [10] when the advanced torque vectoring controller is active, and compares it to the results obtained when no stability controller is active and when the ideal genetic fuzzy yaw moment controller is active. Comparing different parameters of the vehicle response during the double-lanechange maneuver, it can be seen that, although the ATVC has improved all the decisive parameters that describe the handling, stability, and longitudinal dynamics of the vehicle, it cannot be considered as effective as the GFYMC, which represents the ideal case but is not directly realizable. With respect to the handling of the vehicle, the ATVC has reduced $|\beta|_{\max \text { and }}|\dot{\psi}|_{\max }$ by about the same amount as the GFYMC.
The maximum lateral acceleration of the vehicle, $\left|a_{y}\right|_{\max }$, has increased the same amount as it did when the GFYMC was active; however, when the ATVC is active, the driver requires a larger maximum steering wheel angle to negotiate the maneuver. In addition, the hysteresis of the performance plot $\left(\Delta \dot{\psi}_{H}\right)$ is about 1.7 times larger than it is when the GFYMC is active. Altogether, the ATVC is considered to be an effective controller for improving the handling characteristics of the vehicle. Since the ATVC has reduced $|\beta|_{\max \text { and }}|\dot{\psi}|_{\max }$ by about the same amount as the GFYMC, it is considered to be as effective at improving the stability of the vehicle. The speed lost during the maneuver is about the same as that observed when the GFYMC is used and, therefore, the ATVC is also considered to be a very effective controller for improving the longitudinal dynamics of the vehicle. More information about the selection of vehicle states for comparing the effectiveness of different stability control systems can be found in [16].
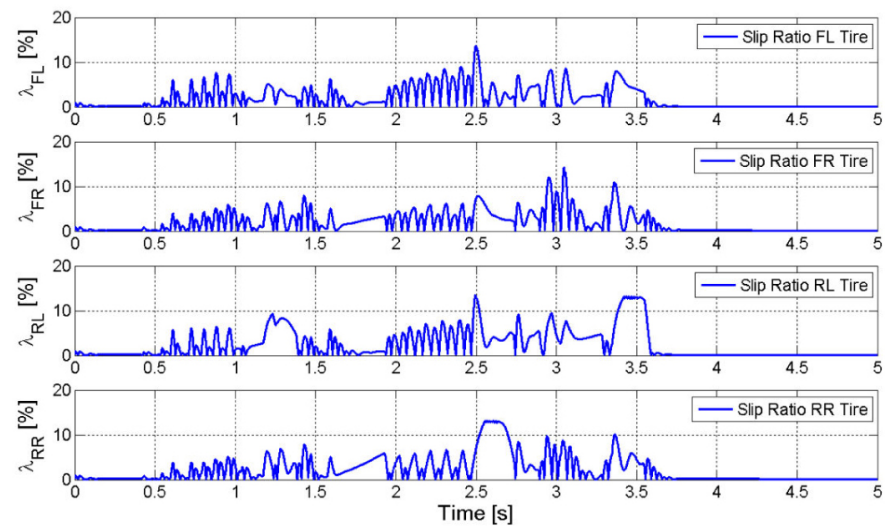

Figure 15. Tire slip ratios when driving through the double-lane-change maneuver using the driver model with the ATVC

Table 2. Vehicle response during the double-lane-change maneuver using the driver model without a controller (AUTO21EV), with the genetic fuzzy yaw moment controller (GFYMC), and with the advanced torque vectoring controller (ATVC)

\begin{tabular}{|c|c|c|c|c|c|c|}
\hline Parameter & $|\beta|_{\max }$ & $|\dot{\psi}|_{\max }$ & $\left|a_{y}\right|_{\max }$ & $\left|\delta_{S W}\right|_{\max }$ & $\Delta \dot{\psi}_{H}$ & $v_{\text {lost }}$ \\
\hline AUTO21EV & $26.3^{\circ}$ & $115.4^{\circ} / \mathrm{s}$ & $8.2 \mathrm{~m} / \mathrm{s}^{2}$ & $545^{\circ}$ & $163.8^{\circ} / \mathrm{s}$ & $20.5 \mathrm{~m} / \mathrm{s}$ \\
\hline GFYMC & $6.2^{\circ}$ & $31.2^{\circ} / \mathrm{s}$ & $8.4 \mathrm{~m} / \mathrm{s}^{2}$ & $108^{\circ}$ & $21^{\circ} / \mathrm{s}$ & $13.2 \mathrm{~m} / \mathrm{s}$ \\
\hline ATVC & $6.1^{\circ}$ & $36.4^{\circ} / \mathrm{s}$ & $8.4 \mathrm{~m} / \mathrm{s}^{2}$ & $140^{\circ}$ & $35.5^{\circ} / \mathrm{s}$ & $13.5 \mathrm{~m} / \mathrm{s}$ \\
\hline
\end{tabular}

\section{Step-Steer Response Maneuver}

To evaluate the performance of the vehicle using the ATVC in a step-steer response maneuver, the vehicle yaw rate, sideslip angle, and lateral acceleration as functions of time are observed. Figure 16 illustrates the steering wheel input and the lateral acceleration of the vehicle. As can be 
seen, the lateral acceleration when using the ATVC is similar to that obtained when using the GFYMC; however, due to the actuation of the in-wheel motors, some small oscillations can be observed in the lateral acceleration plot. The rise time of the lateral acceleration response is about 0.47 seconds when using the ATVC. Figure 17 shows the yaw rate and sideslip angle of the vehicle during this maneuver. A severe oscillation can be observed in the yaw rate response when using the ATVC, which would be perceived by the driver as being disruptive and should be avoided. This result indicates that, in practice, the ATVC should be activated only when the yaw rate error exceeds a particular threshold and should not be used to correct small yaw rate errors. Although the rise time of the yaw rate response is similar to that obtained when using the GFYMC, an overshoot is clearly visible when using the ATVC. The sideslip angle of the vehicle is slightly less than that observed when using the GFYMC but, again, some oscillations can be seen in the response.
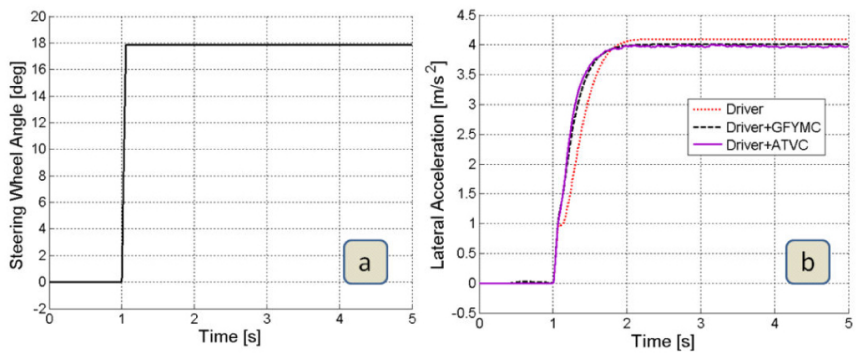

Figure 16. (a) Required steering wheel input and (b) lateral acceleration of the vehicle when driving through the step-steer maneuver using the genetic fuzzy yaw moment controller (GFYMC) and the ATVC
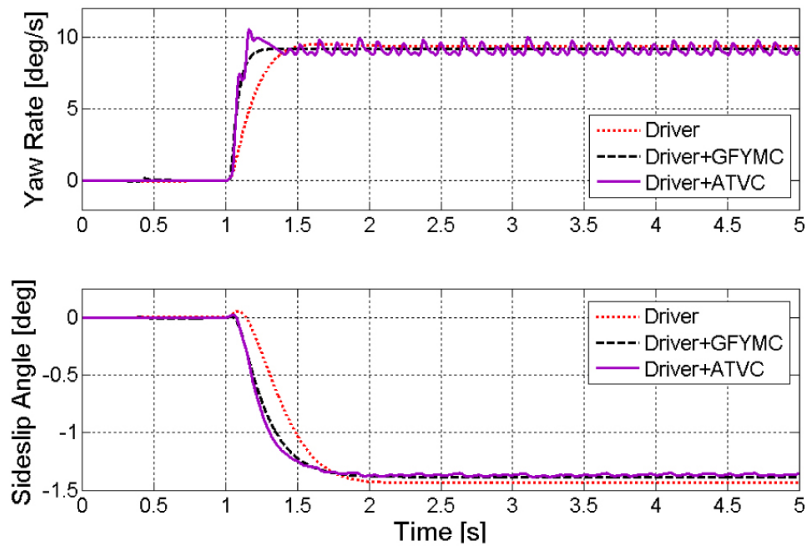

Figure 17. Yaw rate (top) and sideslip angle (bottom) of the vehicle when driving through the step-steer maneuver using the genetic fuzzy yaw moment controller (GFYMC) and the ATVC

Table 3 summarizes the vehicle response during the stepsteer test maneuver. Comparing different parameters of the vehicle response with and without the ATVC, it can be recognized that the ATVC is effective at improving all the decisive performance parameters of the vehicle which, for this maneuver, describe the vehicle handling characteristics. However, two problems must be addressed: the severe oscillation in the yaw rate response of the vehicle and the overshooting effect of the yaw rate response.

Table 3. Vehicle response during the step-steer response maneuver using the driver model without a controller (AUTO21EV), with the genetic fuzzy yaw moment controller (GFYMC), and with the advanced torque vectoring controller (ATVC)

\begin{tabular}{|c|c|c|c|c|}
\hline Parameter & $t_{\dot{\psi}}$ & $\mathrm{PO}=\frac{\dot{\psi}_{\max }-\dot{\psi}_{s s}}{\dot{\psi}_{s s}} \times 100 \%$ & $|\beta|_{\max }$ & $t_{a_{\gamma}}$ \\
\hline AUTO21EV & $0.34 \mathrm{~s}$ & $1.30 \%$ & $1.43^{\circ}$ & $0.66 \mathrm{~s}$ \\
\hline GFYMC & $0.15 \mathrm{~s}$ & $0.00 \%$ & $1.38^{\circ}$ & $0.51 \mathrm{~s}$ \\
\hline ATVC & $0.15 \mathrm{~s}$ & $16.1 \%$ & $1.36^{\circ}$ & $0.47 \mathrm{~s}$ \\
\hline
\end{tabular}

\section{Brake-in-Turn Maneuver}

Figure 18-a illustrates the trajectory of the uncontrolled vehicle relative to the desired path during a brake-in-turn maneuver and compares it to the case when the ATVC is active (Figure 18-b). As can be seen, the vehicle becomes unstable and leaves the predefined road when no stability controller is active. However, the driver model is able to keep the vehicle very close to the predefined circular path while severely braking when the ATVC is active, and the lateral deviation of the vehicle from the desired path remains very small throughout the maneuver.
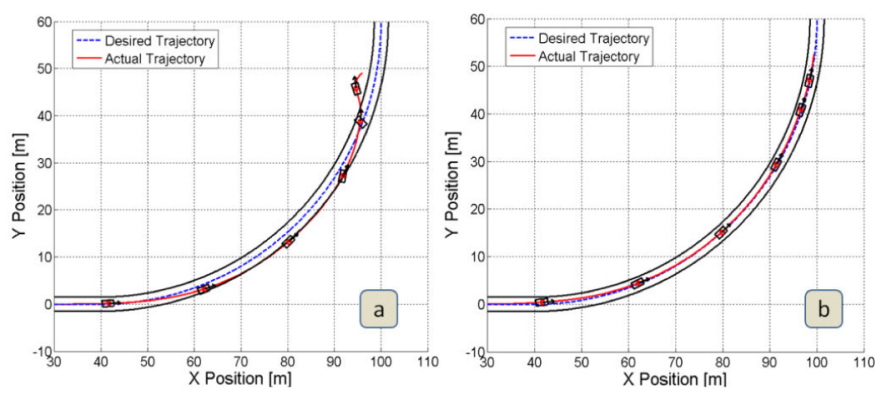

Figure 18. Desired and actual vehicle trajectories when braking in a turn using (a) the driver model only and (b) the driver model with the ATVC

Based on the driver's steering wheel input as a function of time, shown in Figure 19-a, it is clear that the driver model is able to control the vehicle when the ATVC is active by applying a maximum steering wheel angle of only $48^{\circ}$. In addition, the gradient of this plot indicates that it is very easy for the driver to control the vehicle when braking in a turn. Figure 19-b illustrates the lateral acceleration of the vehicle and confirms that the vehicle remains stable when the ATVC is active, even though it is being driven near its handling limit. As can be seen, the lateral acceleration of the vehicle when using the ATVC is very similar to that obtained when 
the GFYMC is active, but contains some oscillations at higher lateral accelerations.
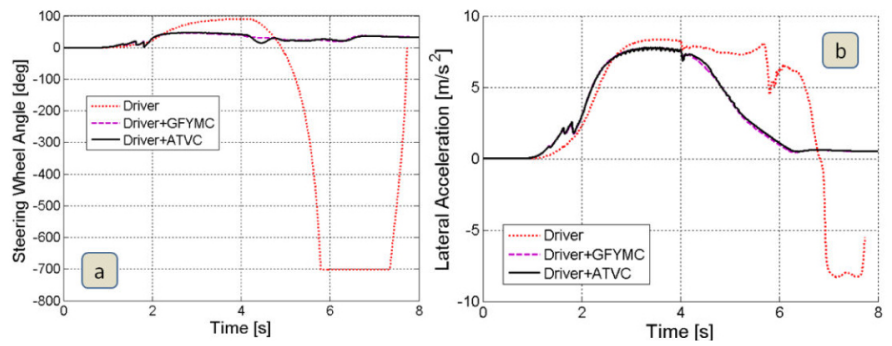

Figure 19. (a) Required steering wheel input and (b) lateral acceleration of the vehicle when braking in a turn using the driver model without a controller, with the genetic fuzzy yaw moment controller (GFYMC), and with the ATVC

Figure 20 illustrates the vehicle yaw rate and sideslip angle when driving through the brake-in-turn maneuver using the GFYMC and ATVC. Note that, for clarity, the yaw rate and sideslip angle of the vehicle are not shown for the case where no stability controller is active. As can be seen, the vehicle yaw rate follows the desired reference model, but it is superimposed with oscillations. In addition, for a short time after the braking starts (between the fourth and fifth seconds of the simulation), the ATVC is not able to minimize the yaw rate error. However, the driver is still able to control the vehicle while braking in the turn. This figure also confirms the stability of the vehicle, since the yaw rate and sideslip angle both approach zero as the vehicle progresses towards larger deceleration rates. Figure 21 shows the vehicle speed as a function of time and the vehicle longitudinal acceleration as a function of vehicle speed. These plots confirm the performance of the speed controller as well as the severity of the braking component of this maneuver.
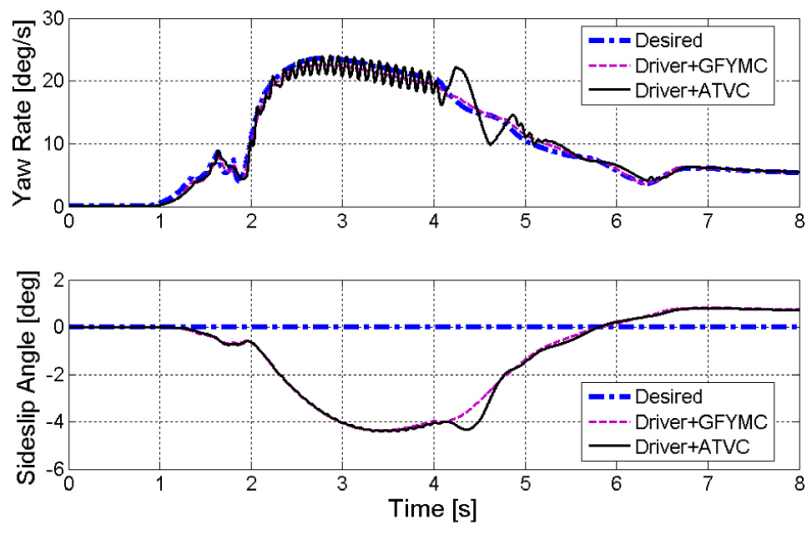

Figure 20. Desired and actual vehicle yaw rate (top) and sideslip angle (bottom) when braking in a turn using the driver model without a controller, with the genetic fuzzy yaw moment controller (GFYMC), and with the ATVC
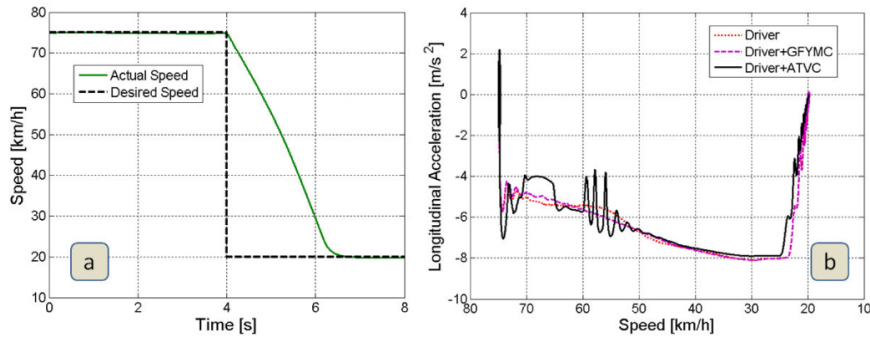

Figure 21. (a) Vehicle speed as a function of time using the driver model with the ATVC, and (b) longitudinal acceleration as a function of vehicle speed when braking

in a turn using the driver model without a controller, with the genetic fuzzy yaw moment controller (GFYMC), and with the ATVC

Table 4 summarizes the vehicle response during the brake-in-turn maneuver using the driver model. Comparing different parameters of the response when this maneuver is performed without a controller, with the GFYMC, and with the ATVC, it can be seen that the ATVC is as effective as the GFYMC at improving all the decisive parameters that describe the handling, stability, and path-following capability of the vehicle during a brake-in-turn maneuver. In particular, the ATVC has reduced $|\beta|_{\max },|\dot{\psi}|_{\max }$, and $\left|\delta_{S W}\right|_{\max }$ substantially, which indicates that this controller is very effective at enhancing the handling capabilities of the vehicle. It is important to notice that, due to the severe oscillations in the yaw rate response of the vehicle (Figure 20), the driver would perceive the activation of the ATVC as being disruptive. A reduction of $|\beta|_{\max \text { and }}|\dot{\psi}|_{\max }$ by the ATVC indicates that it is also very effective at improving the stability of the vehicle. Since the maximum lateral deviation of the vehicle from the desired path remains very small throughout the maneuver, the ATVC is also very effective at enhancing the path-following capability of the vehicle.

Table 4. Vehicle response during the brake-in-turn maneuver using the driver model without a controller (AUTO21EV), with the genetic fuzzy yaw moment controller (GFYMC), and with the advanced torque vectoring controller (ATVC)

\begin{tabular}{|c|c|c|c|c|}
\hline Parameter & $|\dot{\psi}|_{\max }$ & $|\beta|_{\max }$ & $\Delta y_{\max }$ & $\left|\delta_{s W}\right|_{\max }$ \\
\hline AUTO21EV & $126.5^{\circ} / \mathrm{s}$ & $50.1^{\circ}$ & $3.67 \mathrm{~m}$ & $700^{\circ}$ \\
\hline GFYMC & $22.60^{\circ} / \mathrm{s}$ & $4.36^{\circ}$ & $0.18 \mathrm{~m}$ & $46.4^{\circ}$ \\
\hline ATVC & $24.00^{\circ} / \mathrm{s}$ & $4.40^{\circ}$ & $0.18 \mathrm{~m}$ & $48.0^{\circ}$ \\
\hline
\end{tabular}

\section{Straight-Line Braking on a $\mu$-Split Road}

Braking on a $\mu$-split road can be used to confirm the performance and sensitivity of a vehicle and its stability control systems when subjected to external disturbances. Figure 22 shows the vehicle trajectory for this maneuver when no stability controller is active and compares it to the 
case when the ATVC is active. This comparison confirms that the ATVC is able to reduce the side-pushing effect of the vehicle while braking on a $\mu$-split road, but the vehicle still leaves the predefined road, which is considered to be a dangerous situation. The braking distance of the vehicle is reduced to about 47.3 meters when the ATVC is active. From Figure 23, it is clear that the ATVC is able to limit and, later, diminish the yaw rate and sideslip angle of the vehicle while driving over the ice patch, but does not prevent the vehicle from leaving the road.

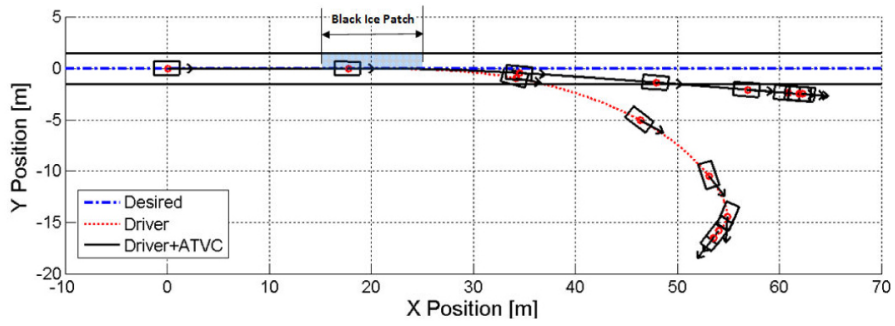

Figure 22. Desired and actual vehicle trajectories when braking on a $\mu$-split road holding the steering wheel fixed with and without the ATVC
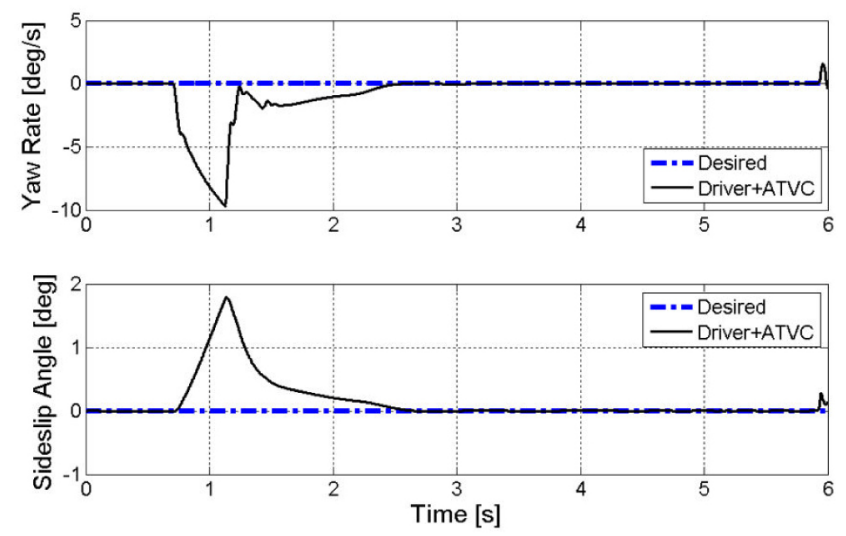

Figure 23. Desired and actual vehicle yaw rate (top) and sideslip angle (bottom) when braking on a $\mu$-split road holding the steering wheel fixed with and without the ATVC

Figure 24 illustrates the tire slip ratios while braking on a $\mu$-split road, and indicates that the slip controllers on the left wheels have limited the motor torques between 0.7 and 1.15 seconds of the simulation in order to prevent the tires from locking up while, at the same time, ensuring the maximum possible braking force is being applied when braking on the ice patch. Later in the simulation, due to the weight shift to the front axle, the slip controllers on the rear axle have limited the motor torques to prevent tire lock-up at higher deceleration rates. Looking at Figure 25, which illustrates the motor torques of all four wheels, it is apparent that the slip controllers on the left wheels have limited the braking torques to $20 \mathrm{Nm}$ (between 0.7 and 1.15 seconds) in order to prevent tire lock-up when braking on the ice patch. As mentioned earlier, as a result of the asymmetric braking forces generated on the left and right wheels, the vehicle is pushed to the right side of the road. In order to prevent this side-pushing effect, the left-to-right torque vectoring controller has requested larger braking forces on the left wheels, which are restricted by the slip controllers [9], and has reduced the braking forces on the right wheels.
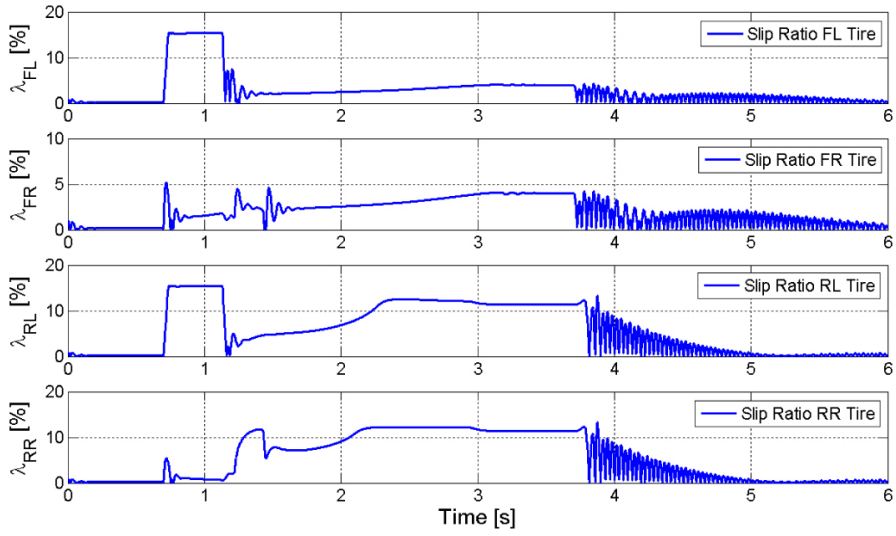

Figure 24. Tire slip ratios when braking on a $\mu$-split road holding the steering wheel fixed and using the ATVC

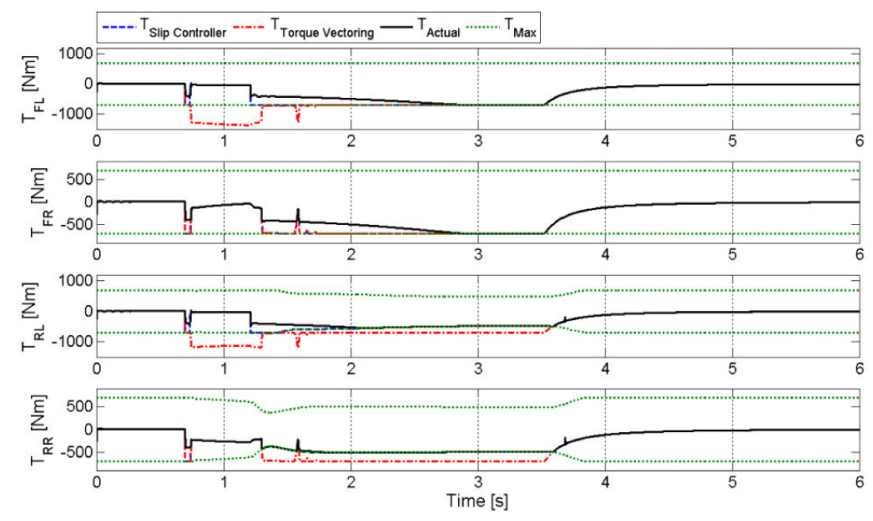

Figure 25. Requested and actual motor torques at each wheel when braking on a $\mu$-split road holding the steering wheel fixed and using the ATVC

Figure 26 illustrates the torque distribution requested by the front-to-rear torque vectoring controller. As can be seen, the front-to-rear torque vectoring controller has requested the front motors to generate up to $60 \%$ of the required corrective yaw moment in order to correct the undesirable side-pushing effect when driving over the ice patch. Requesting more torque from the front motors reduces the lateral force potential of the front axle and increases that of the rear axle. The asymmetric lateral force potentials on the front and rear axles helps to generate the required corrective yaw moment.

Table 5 summarizes the vehicle response during the straight-line braking on a $\mu$-split road maneuver when using the ATVC. Comparing different parameters of the vehicle response during this maneuver, it can be seen that the 


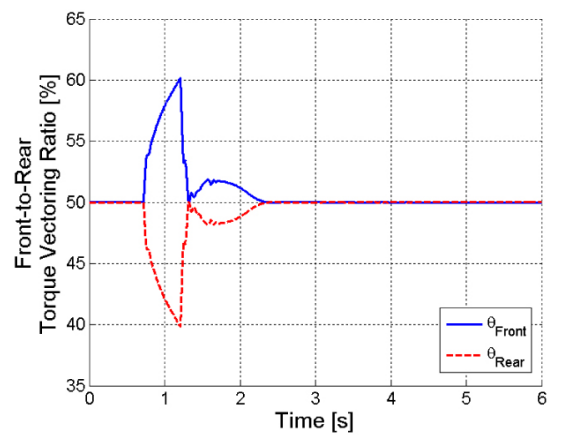

Figure 26. Front-to-rear torque vectoring activation when braking on a $\mu$-split road holding the steering wheel fixed and using the ATVC

effectiveness of the ATVC at improving the stability, pathfollowing capability, and braking performance of the vehicle is limited. Although the ATVC has reduced the $|\beta|_{\max }$ and $|\dot{\psi}|_{\max }$ values in comparison to those obtained when no stability controller was active and has avoided instability, its intervention was not large enough to prevent the vehicle from leaving the predefined road. Moreover, when the ATVC is active, the braking distance of the vehicle is longer than that observed when the GFYMC is active. Finally, the ATVC could not keep the vehicle on the predefined road, which indicates that the ATVC cannot be considered an effective controller for enhancing the path-following capability of the vehicle when braking on a $\mu$-split road.

Table 5. Vehicle response during the straight-line braking on a $\mu$-split road maneuver holding the steering wheel fixed without a controller (AUTO21EV), with the genetic fuzzy yaw moment controller (GFYMC), and with the advanced torque vectoring controller (ATVC)

\begin{tabular}{|c|c|c|c|c|}
\hline Parameter & $|\beta|_{\max }$ & $|\dot{\psi}|_{\max }$ & $\Delta x_{\text {braking }}$ & $\Delta y_{\max }$ \\
\hline AUTO21EV & $24.2^{\circ}$ & $63.4^{\circ} / \mathrm{s}$ & $48.4 \mathrm{~m}$ & $15.9 \mathrm{~m}$ \\
\hline GFYMC & $0.40^{\circ}$ & $1.85^{\circ} / \mathrm{s}$ & $44.5 \mathrm{~m}$ & $0.46 \mathrm{~m}$ \\
\hline ATVC & $1.70^{\circ}$ & $9.70^{\circ} / \mathrm{s}$ & $47.3 \mathrm{~m}$ & $2.70 \mathrm{~m}$
\end{tabular}

\section{EVALUATION USING A DRIVING SIMULATOR}

A hardware-in-the-loop (HIL) and operator-in-the-loop (OIL) driving simulator has been used to confirm the effectiveness of the proposed advanced torque vectoring controller (ATVC) in a more realistic simulation environment. A photograph of the driving simulator used in this work is shown in Figure 27. The vehicle model has been implemented on one core of a quad-core real-time computer. To guarantee real-time performance, the vehicle is modeled with a torque driver applied to each wheel, and the four inwheel motor models have been implemented on a separate core. The torques generated by the motors are transmitted to the first core at each time step of the simulation. The slip controllers and advanced torque vectoring control system have been implemented on the third core, receiving sensor signals and broadcasting control signals at regular intervals. The fourth core is reserved for communication with external devices, including the Windows-based laptop, which provides graphical feedback and receives inputs from the human driver through a steering wheel and pedals. The performance of the ATVC is evaluated on the driving simulator using the ISO double-lane-change maneuver, the brake-in-turn maneuver, and braking on a $\mu$-split road, all of which have been described above.

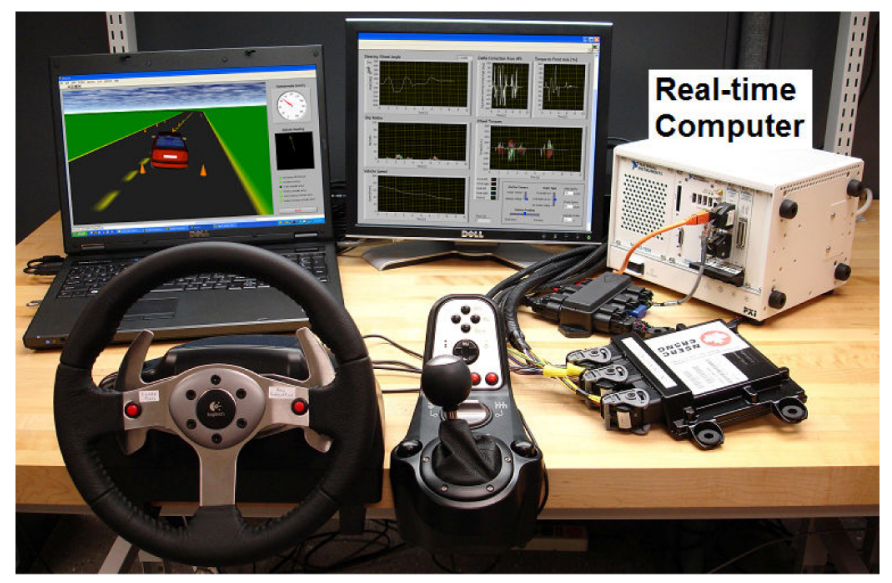

Figure 27. Driving simulator used to further evaluate the performance of the advanced torque vectoring controller (ATVC)

\section{ISO Double-Lane-Change Maneuver}

We first consider the double-lane-change maneuver described earlier; however, the initial vehicle speed is reduced to $60 \mathrm{~km} / \mathrm{h}$ to make this maneuver feasible for a nonprofessional human driver. Even with this modification, the maneuver was found to be quite challenging without the assistance of the ATVC. When aided by the controller, the maneuver required substantially less effort to complete. As shown in Figure 28-a, the maneuver was successfully completed both with and without the controller; however, when the controller was activated, substantially less steering 
effort was required by the driver, as shown in Figure 28-b. Note, in particular, the considerable steering effort required to recover from the second lane change without the controller (between 4 and 4.5 seconds of the simulation) compared to that required when assisted by the controller. Also note that the driver was required to anticipate the second lane change without the controller, initiating the change in steering wheel angle about 0.5 seconds earlier than was necessary when assisted by the ATVC.
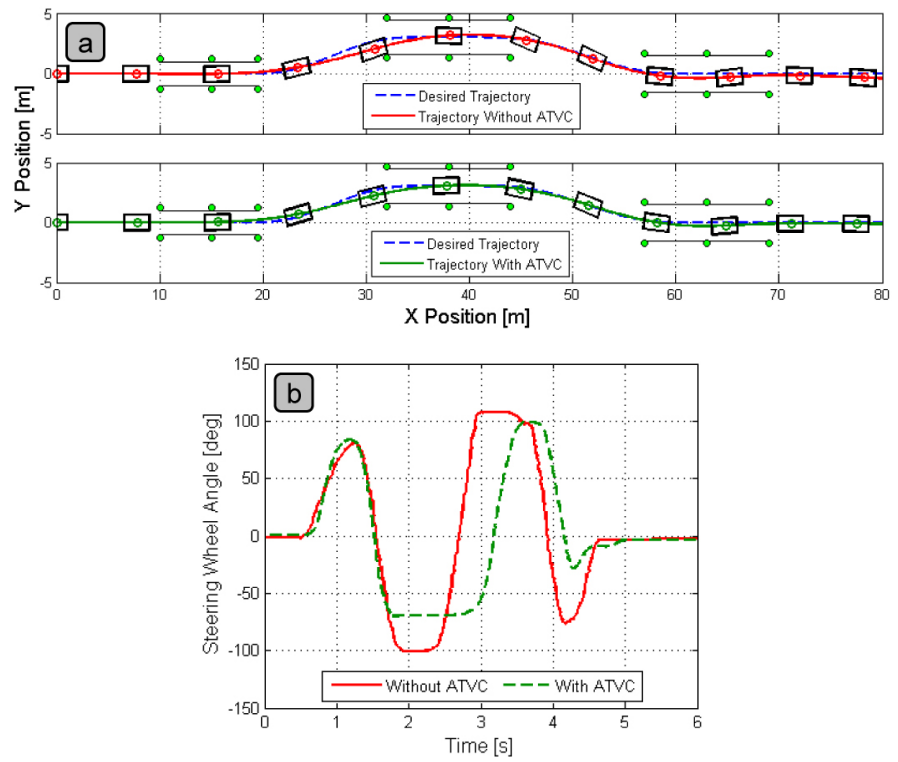

Figure 28. Vehicle trajectory (a) and steering wheel angle provided by the driver (b) during the double-lanechange maneuver performed on the driving simulator with and without the ATVC

Figure 29-a shows the same driving performance metric as shown in Figure 11-d, once again indicating that the steering performance of the vehicle is substantially improved by the ATVC. The desired yaw rate and actual yaw rates with and without the controller are shown in Figure 29-b, and closely match the previous results shown in Figure 10. Note that the oscillations in the yaw rate plot are substantially smaller in the driving simulator results due to the realistic communication delays between the vehicle model and controller, and due to the rate at which data is collected from the simulator. Finally, the front-to-rear torque distribution ratio and the torques applied to each wheel are shown in Figures 29-c and 29-d, respectively, where the four main phases of steering (enter left lane, exit left lane and enter right lane, complete second lane change, and resume driving straight) are readily apparent.
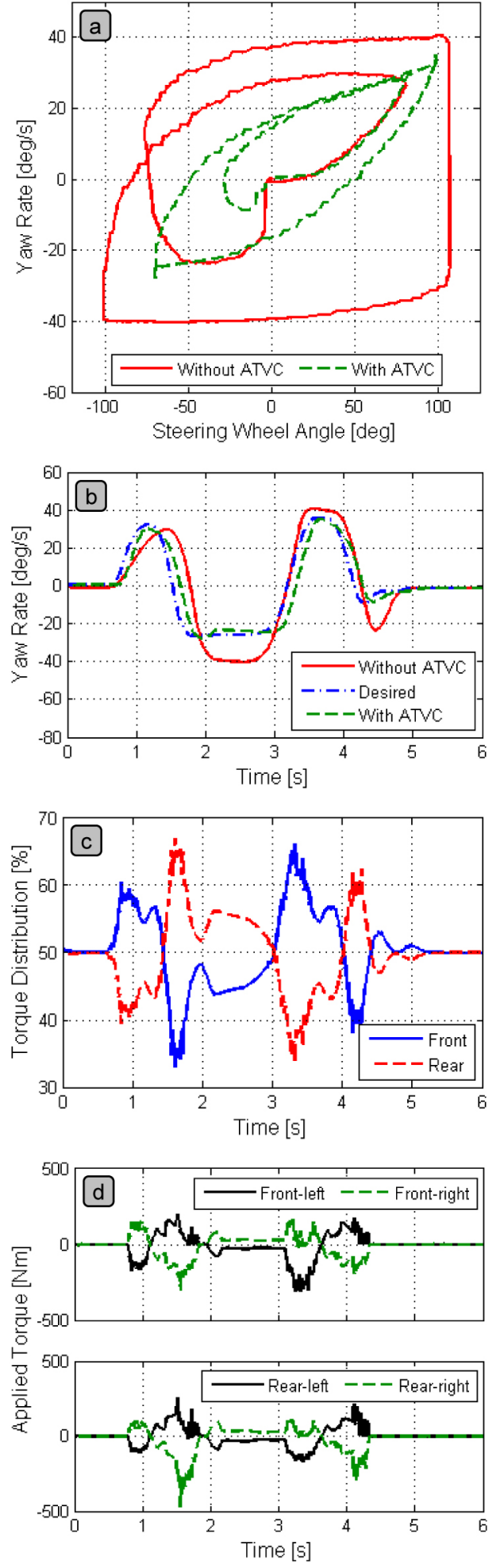

Figure 29. Vehicle yaw rate as a function of driver steering wheel input (a) and time (b), front-to-rear torque distribution ratio (c), and applied wheel torques (d) during the double-lane-change maneuver performed on the driving simulator 


\section{Brake-in-Turn Maneuver}

We now consider the brake-in-turn maneuver. As before, the vehicle enters a curve of radius $60 \mathrm{~m}$ traveling at a constant speed. Once a steady-state yaw rate has been reached, the speed is decreased to $20 \mathrm{~km} / \mathrm{h}$. To make this maneuver feasible for a non-professional human driver, the initial speed is set to $70 \mathrm{~km} / \mathrm{h}$ (Figure 30-a). As shown in Figures 30-b and $\underline{30-c}$, the maneuver was successfully completed both without and with the controller, respectively. Qualitatively, the driver required more concentration to complete the maneuver without the assistance of the ATVC. A quantitative measure of the steering effort required by the driver is shown in Figure 31-a. Although some countersteering was used in both cases, the ATVC clearly reduced both the amplitude and the frequency thereof, indicating that the maneuver was more taxing on the driver when the ATVC was absent. The front-to-rear torque distribution ratio and the torques applied to each wheel are shown in Figures 31-b and $\underline{31-c}$, respectively. Note that the ATVC provides assistance in normal driving conditions (between 2.5 and 5 seconds) as well as more extreme situations (between 5 and 7 seconds).
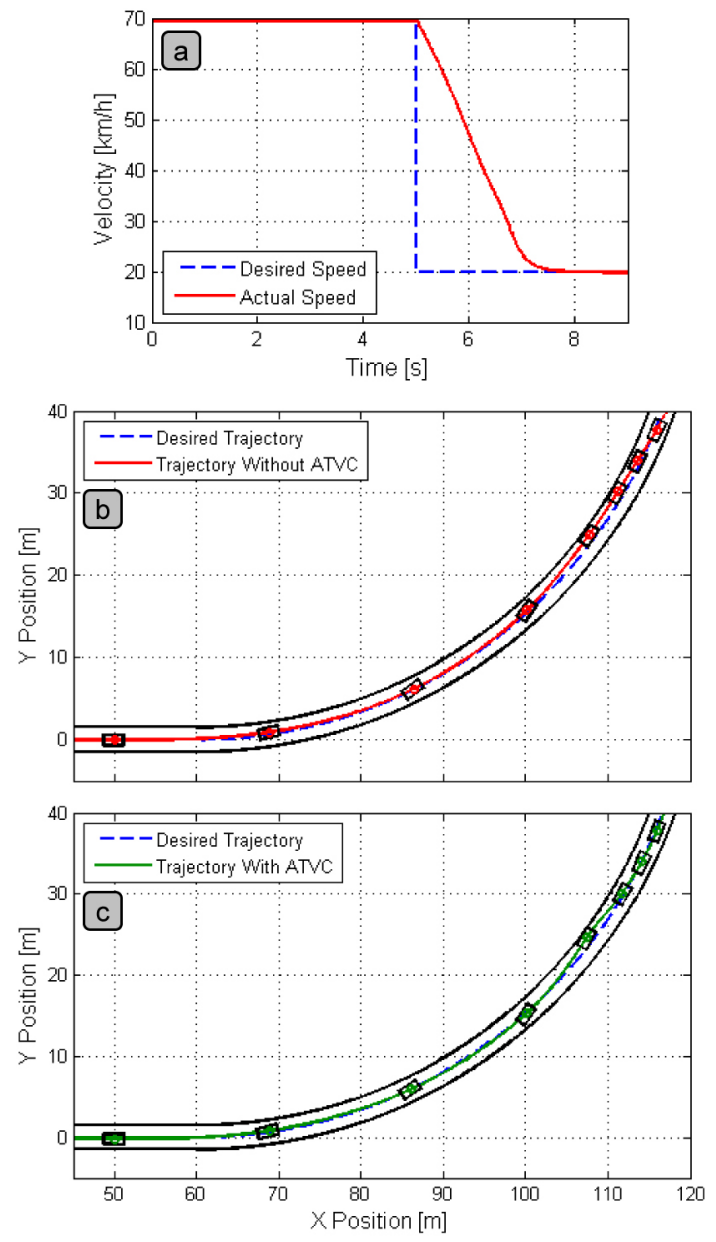

Figure 30. Vehicle speed (a) and trajectories without (b) and with (c) the ATVC during the brake-in-turn maneuver performed on the driving simulator
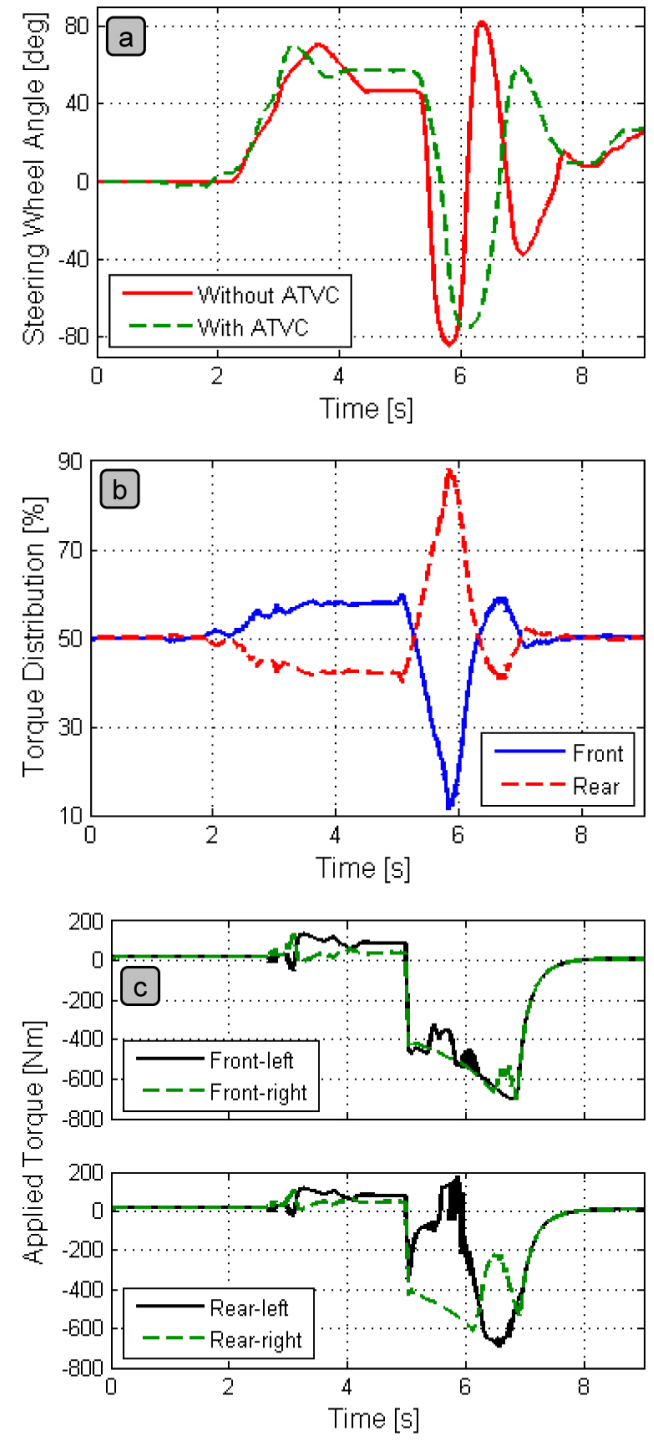

Figure 31. Steering wheel angle provided by the driver (a), front-to-rear torque distribution ratio (b), and applied wheel torques (c) during the brake-in-turn maneuver performed on the driving simulator

\section{Straight-Line Braking on a $\mu$-Split Road}

The final maneuver we evaluate using the driving simulator is straight-line braking on a $\mu$-split road. As shown in Figure 32-a , the vehicle is braked from a speed of $80 \mathrm{~km} / \mathrm{h}$ to $5 \mathrm{~km} / \mathrm{h}$ while passing over a patch of ice on the left half of the road. The trajectories of the vehicle with and without the ATVC are shown in Figure 32-b. Once again, we find that the ATVC is unable to prevent the vehicle from leaving the road; however, the deviation from the desired straight-line trajectory is reduced and, as shown in Figure 33-a, the stability of the vehicle is improved substantially. Furthermore, since the ATVC restores the stability and maneuverability of the vehicle, a human driver or an active steering controller can easily correct the trajectory when the 
ATVC is active. As shown in Figures 33-b and 33-c, more net torque is distributed to the front axle during this maneuver, which agrees with the results obtained previously (Figure 26).
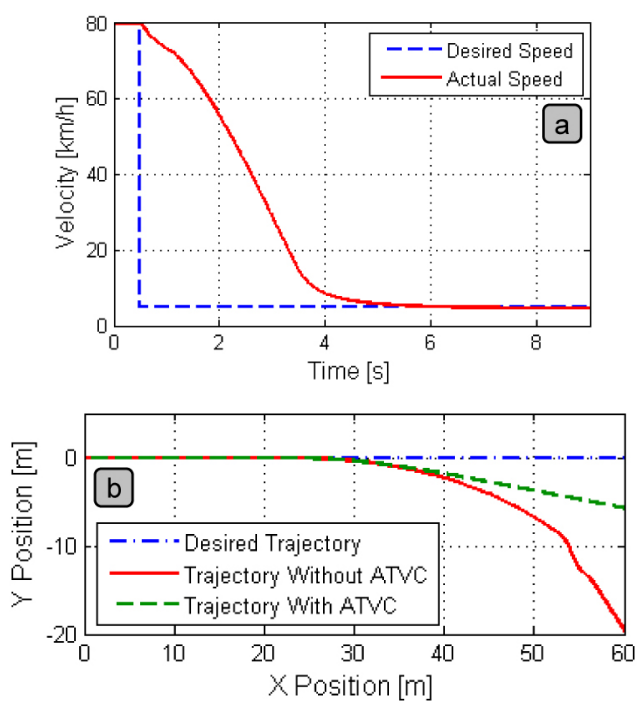

Figure 32. Vehicle speed (a) and trajectories with and without the ATVC (b) while braking on a $\mu$-split road using the driving simulator
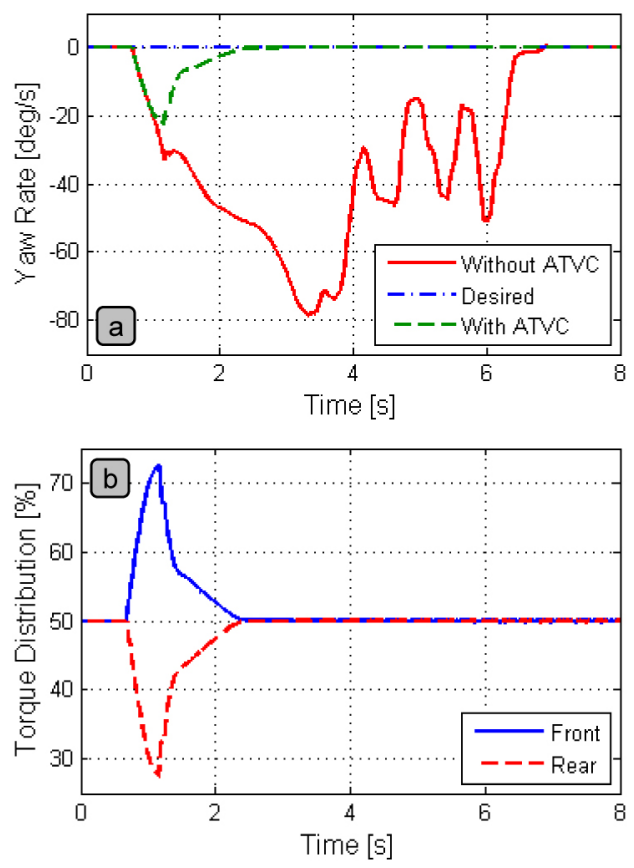

Figure 33.

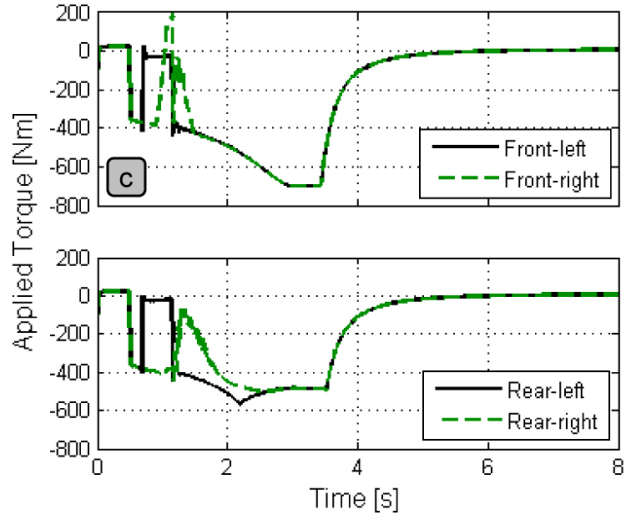

Figure 33. (cont.) Yaw rate (a), front-to-rear torque distribution ratio (b), and applied wheel torques (c) while braking on a $\mu$-split road using the driving simulator

\section{CONCLUSIONS}

In this work, an advanced torque vectoring controller is developed based on the genetic fuzzy yaw moment controller developed previously. The objective of the advanced torque vectoring controller is to distribute the calculated corrective yaw moment to the individual in-wheel motors in order to stabilize the vehicle driving dynamics. A novel algorithm is developed for the left-to-right torque vectoring on each axle, and a proportional-derivative controller is introduced for the front-to-rear torque vectoring distribution action. Several maneuvers are performed in pure simulation and using an operator-in-the-loop driving simulator to demonstrate the performance and effectiveness of the advanced torque vectoring controller.

\section{ACKNOWLEDGMENTS}

Funding for this work was provided by the Natural Sciences and Engineering Research Council of Canada and a grant from AUTO21, a Canadian Network of Centres of Excellence.

\section{REFERENCES}

1. Wheals, J.C.: "Torque vectoring center differential for AWD: Design and integration", Innovative Fahrzeug-Getriebe Symposium, IIR Deutschland GmbH, 2002.

2. Wheals, J., "Torque Vectoring Driveline: SUV-based Demonstrator and Practical Actuation Technologies," SAE Technical Paper 2005-01-0553, 2005, doi: 10.4271/2005-01-0553

3. Pelchen, C., Zdych, R., Baasch, D., and Kubalczyk, R.: "Improvement of vehicle agility and safety by means of wheel torque based driving dynamics", 14th Aachen Colloquium, Automobile and Engine Technology, 2005

4. Wunschelmeier, U. and Huchtkoetter, H.: "Traction and stability enhancement using active limited-slip differentials", 14th Aachen Colloquium, Automobile and Engine Technology, 2005.

5. Wheals, J., Baker, H., Ramsey, K., and Turner, W., "Torque Vectoring AWD Driveline: Design, Simulation, Capabilities and Control," SAE Technical Paper 2004-01-0863, 2004, doi: 10.4271/2004-01-0863.

6. Jalali, K.: The Concept and Development of an Optimized Actuator for the Clutch of a Transfer Case, Diploma Thesis, RWTH Aachen, 2004.

7. Reimpell, J., Stoll, H., and Betzler, J., "The Automotive Chassis Engineering Principles," Society of Automotive Engineers, Inc. and 
Butterworth Heinemann, Warrendale, PA, ISBN 978-0-7680-0657-5, 2001.

8. Wallentowitz, H. and Reif, K.: Handbuch Kraftfahrzeugelektronik: Grundlagen, Komponenten, Systeme, Anwendungen, ATZ/MTZFachbuch, Friedr. Vieweg \& Sohn Verlag, 2006.

9. Jalali, K., Uchida, T., McPhee, J., and Lambert, S., "Development of a Fuzzy Slip Control System for Electric Vehicles with In-wheel Motors," SAE Int. J. Alt. Power. 1(1):46-64, 2012, doi: 10.4271/2012-01-0248.

10. Jalali, K., Lambert, S., and McPhee, J., "Development of a Pathfollowing and a Speed Control Driver Model for an Electric Vehicle," SAE Int. J. Passeng. Cars - Electron. Electr. Syst. 5(1):100-113, 2012, doi: $10.4271 / 2012-01-0250$.

11. Jalali, K., Uchida, T., McPhee, J., and Lambert, S., "Integrated Stability Control System for Electric Vehicles with In-wheel Motors using Soft Computing Techniques," SAE Int. J. Passeng. Cars - Electron. Electr. Syst. 2(1):109-119, 2009, doi: 10.4271/2009-01-0435.

12. Pacejka, H., "Tire and Vehicle Dynamics," Society of Automotive Engineers, Inc. and Butterworth Heinemann, Warrendale, PA, ISBN 978-0-7680-1126-5, 2002.

13. Pacejka, H.B. and Besselink, I.J.M.: "Magic formula tyre model with transient properties", Vehicle System Dynamics, 27(Suppl.):234-249, 1997.

14. Wallentowitz, H.: Vertical and Lateral Dynamics of Passenger Vehicles, Automotive Technology II, ("Vertical- und Querdynamik von Kraftfahrzeugen, Voerlesungsumdruck Fahrzeugtechnik II"), course notes, Institute of Automotive Engineering, Aachen University of Technology, Germany, 2005.

15. Kiencke, U. and Nielsen, L.: Automotive Control Systems for Engine, Driveline, and Vehicle, Springer, 2nd edition, 2005.

16. Jalali, K.: Stability Control of Electric Vehicles with In-wheel Motors: A New Approach Using Soft Computing Techniques, LAP LAMBERT Academic Publishing, 2012. 